\title{
Rate-limiting Steps in The Intramolecular C-H Activation of Ruthenium N-Heterocyclic Carbene Complexes
}

\author{
Katarzyna Młodzikowska-Pieńko *† and Bartosz Trzaskowski *†
}

†Centre of New Technologies, University of Warsaw, Banacha 2C St., 02-097 Warsaw

¥Faculty of Chemistry, University of Warsaw, Pasteura 1 St., 02-093 Warsaw

Emails: Katarzyna Młodzikowska-Pieńko - k.mlodzikowska@cent.uw.edu.pl

Bartosz Trzaskowski - trzask@cent.uw.edu.pl

\section{Contents}

Scheme S1 M06 and CCSD-DLPNO Gibbs free energy profile for the ethylene hydrogenation for catalyst 1 .

Table S1 Total energy values and Gibbs free energy values ( $\Delta \mathrm{G}$ as definied in the manuscript) for catalysts $3,4,5,6$ and its complexes.

Table S2 Gibbs free energy profile for the ethylene hydrogenation for catalysts $\mathbf{3}, \mathbf{4}, \mathbf{5}, \mathbf{6}$. Data obtained using rigid rotor approximation.

Table S3 Thermochemistry data obtained using rigid rotor approximation for catalysts $\mathbf{3}, \mathbf{4}, \mathbf{5}$, 6.

2 Table S4 Total energy value(E) and Gibbs free energy values ( $\Delta \mathrm{G}$ as definied in the manuscript) for catalysts 1 and its complexes.

Table S5 The F-SAPT and SAPT0 contribution for transition states ts5, ts7 and ts9 for catalysts $3,4,5,6$.

3 Table S6 The RDG effective density plots for intermediate int 3 and transition state ts5, ts7, ts9 for every considered catalysts $(\mathbf{3}, \mathbf{4}, \mathbf{5}, \mathbf{6})$.

4 Table S7 The critical points (CPs) $(3,-1)$ and its characterization for intermediate int3 and transition state ts5, ts 7 , ts 9 for every considered catalysts $(\mathbf{3}, \mathbf{4}, \mathbf{5}, \mathbf{6})$.

5 Table S8 The critical points $(\mathrm{CPs})(3,+1)$ and its characterization for intermediate int3 and transition state ts5, ts 7 , ts 9 for every considered catalysts $(\mathbf{3}, \mathbf{4}, \mathbf{5}, \mathbf{6})$.

References 


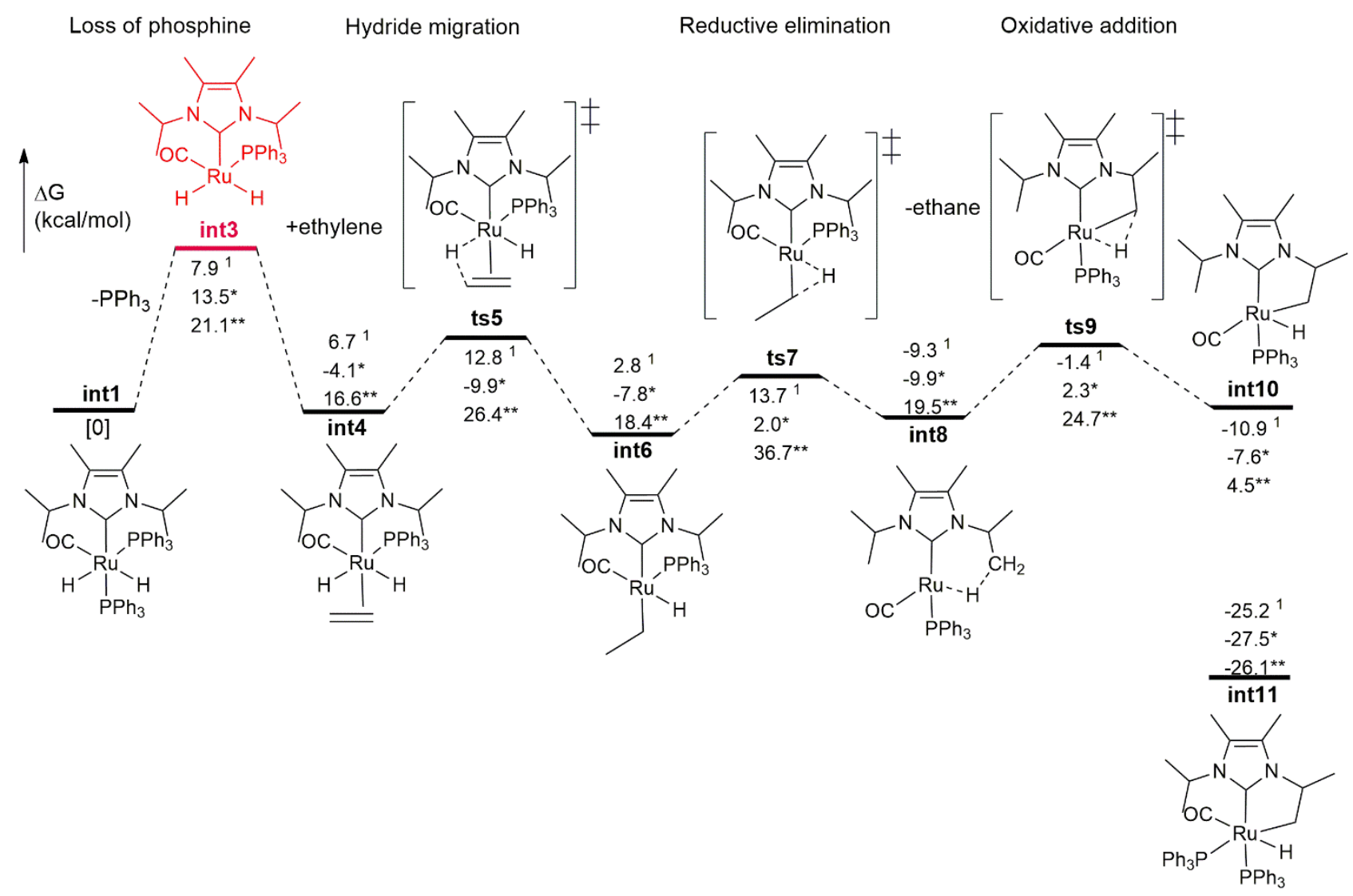

Scheme S1. Gibbs free energy profile for catalyst 1. ${ }^{1}$ Values obtained by Houk et al. ${ }^{1},{ }^{*}$ values obtained from M06 method (basis set LACVP++**), ${ }^{* *}$ values obtained from DLPNO-CCSD(T) (basis set def2-svp/ def2/J)approach.

Table S1. Total energy values (E) and Gibbs free energy values (G; as defined in the manuscript) for catalyst $(\mathbf{3}, \mathbf{4}, \mathbf{5}, \mathbf{6})$ and its complexes.

\begin{tabular}{|c|c|c|c|c|c|c|c|}
\hline Structure & $\begin{array}{c}\text { M06 E. (H) } \\
(E)\end{array}$ & $\begin{array}{c}\text { Solvatation } \mathbf{E} \\
\text { in toluene }\end{array}$ & $\begin{array}{c}\text { Zero point } \\
\text { energy } \\
\text { (kcal/mol) }\end{array}$ & $\begin{array}{c}\text { Entropy } \\
\left(\mathrm{cal} /\left(\mathrm{mol}^{*} \mathrm{~K}\right)\right)\end{array}$ & $\begin{array}{c}\text { Thermal } \\
\text { correction to } \\
\text { enthalpy (H) }\end{array}$ & M06 G (H) & $\begin{array}{c}\text { D3 correction } \\
\text { (H) }\end{array}$ \\
\hline int1-3 & -2743.388 & 0.0018 & 523.1 & 293.1 & 0.052 & -2742.639 & -0.038 \\
\hline int3-3 & -1707.416 & 0.0002 & 351.6 & 219.9 & 0.036 & -1706.924 & -0.019 \\
\hline int4-3 & -1785.985 & 0.0023 & 385.0 & 235.6 & 0.040 & -1785.441 & -0.021 \\
\hline ts5-3 & -1785.975 & 0.0020 & 384.6 & 228.7 & 0.039 & -1785.429 & -0.021 \\
\hline int6-3 & -1785.991 & 0.0014 & 386.3 & 239.8 & 0.040 & -1785.447 & -0.021 \\
\hline ts $7-3$ & -1785.974 & 0.0015 & 385.6 & 238.4 & 0.040 & -1785.431 & -0.022 \\
\hline int8-3 & -1706.220 & 0.0008 & 341.7 & 212.4 & 0.035 & -1705.741 & -0.017 \\
\hline ts9-3 & -1706.195 & 0.0017 & 338.3 & 212.3 & 0.035 & -1705.720 & -0.017 \\
\hline int10-3 & -1706.212 & 0.0020 & 339.1 & 213.0 & 0.035 & -1705.736 & -0.017 \\
\hline int11-3 & -2742.196 & 0.0022 & 511.6 & 289.8 & 0.052 & -2741.464 & -0.038 \\
\hline ethylene & -78.516 & 0.0028 & 31.8 & 52.4 & 0.004 & -78.484 & -0.0001 \\
\hline ethane & -79.758 & 0.0042 & 46.7 & 58.0 & 0.004 & -79.702 & -0.0001 \\
\hline phosphine & -1035.911 & 0.0024 & 170.7 & 130.1 & 0.017 & -1035.681 & -0.0062 \\
\hline int1-4 & -2742.194 & 0.0019 & 508.6 & 299.2 & 0.053 & -2741.470 & -0.037 \\
\hline int3-4 & -1706.216 & -0.0009 & 336.6 & 218.1 & 0.036 & -1705.748 & -0.017 \\
\hline int4-4 & -1784.787 & 0.0015 & 370.6 & 228.7 & 0.038 & -1784.264 & -0.020 \\
\hline ts5-4 & -1784.774 & 0.0012 & 369.9 & 226.0 & 0.038 & -1784.252 & -0.020 \\
\hline
\end{tabular}




\begin{tabular}{|c|c|c|c|c|c|c|c|}
\hline int6-4 & -1784.789 & 0.0009 & 371.8 & 228.5 & 0.038 & -1784.266 & -0.020 \\
\hline ts 7-4 & -1784.774 & 0.0007 & 371.3 & 232.2 & 0.039 & -1784.253 & -0.021 \\
\hline int8-4 & -1705.022 & -0.0009 & 327.2 & 212.2 & 0.035 & -1704.568 & -0.016 \\
\hline ts9-4 & -1704.997 & 0.0003 & 324.0 & 211.7 & 0.034 & -1704.547 & -0.016 \\
\hline int10-4 & -1705.015 & 0.0010 & 324.7 & 212.4 & 0.035 & -1704.563 & -0.016 \\
\hline int11-4 & -2740.991 & 0.0015 & 496.7 & 291.3 & 0.052 & -2740.284 & -0.037 \\
\hline int1-5 & -2975.552 & 0.0029 & 591.2 & 311.6 & 0.056 & -2974.698 & -0.044 \\
\hline int3-5 & -1939.562 & 0.0017 & 418.7 & 231.3 & 0.038 & -1938.965 & -0.022 \\
\hline int4-5 & -2018.141 & 0.0033 & 453.3 & 246.0 & 0.042 & -2017.490 & -0.026 \\
\hline ts5-5 & -2018.127 & 0.0028 & 452.7 & 241.3 & 0.041 & -2017.476 & -0.025 \\
\hline int6-5 & -2018.143 & 0.0022 & 454.4 & 246.4 & 0.042 & -2017.491 & -0.025 \\
\hline ts7-5 & -2018.127 & 0.0022 & 453.9 & 250.2 & 0.042 & -2017.478 & -0.026 \\
\hline int8-5 & -1938.372 & 0.0015 & 409.5 & 229.0 & 0.038 & -1937.789 & -0.021 \\
\hline ts9-5 & -1938.348 & 0.0025 & 406.2 & 223.1 & 0.037 & -1937.767 & -0.022 \\
\hline int10-5 & -1938.353 & 0.0029 & 407.1 & 226.1 & 0.038 & -1937.771 & -0.021 \\
\hline int11-5 & -2974.342 & 0.0025 & 579.6 & 300.8 & 0.055 & -2973.503 & -0.045 \\
\hline int1-6 & -3204.058 & 0.0021 & 606.3 & 325.4 & 0.060 & -3203.183 & -0.050 \\
\hline int3-6 & -2168.083 & 0.0019 & 433.7 & 255.6 & 0.043 & -2167.467 & -0.027 \\
\hline int4-6 & -2246.656 & 0.0018 & 467.6 & 269.3 & 0.047 & -2245.990 & -0.031 \\
\hline ts5-6 & -2246.637 & 0.0015 & 467.4 & 263.3 & 0.046 & -2245.970 & -0.030 \\
\hline int6-6 & -2246.649 & 0.0014 & 468.6 & 265.0 & 0.046 & -2245.980 & -0.031 \\
\hline ts7-6 & -2246.636 & 0.0013 & 469.1 & 258.0 & 0.045 & -2245.964 & -0.031 \\
\hline int8-6 & -2166.881 & 0.0014 & 424.1 & 258.2 & 0.043 & -2166.282 & -0.026 \\
\hline ts9-6 & -2166.861 & 0.0010 & 421.6 & 246.0 & 0.042 & -2166.262 & -0.026 \\
\hline int10-6 & -2166.881 & 0.0013 & 422.9 & 248.3 & 0.042 & -2166.281 & -0.026 \\
\hline int11-6 & -3202.864 & 0.0016 & 595.3 & 321.5 & 0.059 & -3202.006 & -0.049 \\
\hline
\end{tabular}

Table S2. Gibbs free energy profile for the ethylene hydrogenation for catalysts 3, 4, 5, 6. $\Delta \mathbf{G}$-values obtained using thermochemistry data from (M06-D3/LACVP++**) approach (Jaguar software); $\Delta$ G2- values obtained using thermochemistry data obtained using rigid rotor approximation Grimme's quasirrho; $\Delta$ G3- values obtained using thermochemistry data obtained using rigid rotor Cramer and Truhlar's quasi-harmonic approximation.

\begin{tabular}{|c|c|c|c|}
\hline Structure & $\Delta G$ & $\Delta \mathbf{G} 2$ & $\Delta \mathbf{G} 3$ \\
\hline int1-3 & 0.0 & 0.0 & 0.0 \\
\hline int3-3 & 21.4 & 23.0 & 22.6 \\
\hline int4-3 & 0.4 & 2.7 & 1.6 \\
\hline ts5-3 & 7.7 & 9.0 & 7.9 \\
\hline int6-3 & -3.4 & -1.0 & -2.0 \\
\hline ts 7-3 & 6.5 & 8.4 & 7.5 \\
\hline int8-3 & -0.9 & 0.3 & -0.5 \\
\hline ts9-3 & 12.4 & 13.7 & 12.7 \\
\hline int10-3 & 2.5 & 3.8 & 2.7 \\
\hline int11-3 & -27.0 & -25.6 & -26.8 \\
\hline int1-4 & 0.0 & 0.0 & 0.0 \\
\hline int3-4 & 25.6 & 24.5 & 24.5 \\
\hline int4-4 & 4.8 & 3.7 & 3.2 \\
\hline ts5-4 & 12.3 & 11.0 & 10.5 \\
\hline int6-4 & 3.8 & 1.9 & 1.5 \\
\hline ts 7-4 & 12.3 & 11.0 & 10.7 \\
\hline int8-4 & 1.6 & 0.6 & 0.3 \\
\hline ts9-4 & 14.8 & 14.2 & 13.8 \\
\hline int10-4 & 4.7 & 3.8 & 3.4 \\
\hline int11-4 & -20.4 & -20.5 & -20.7 \\
\hline int1-5 & 0.0 & 0.0 & 0.0 \\
\hline int3-5 & 32.9 & 31.2 & 32.0 \\
\hline int4-5 & 6.9 & 6.2 & 6.2 \\
\hline ts5-5 & 15.4 & 14.2 & 14.2 \\
\hline int6-5 & 5.7 & 4.4 & 4.7 \\
\hline ts 7-5 & 14.4 & 13.7 & 14.1 \\
\hline int8-5 & 6.3 & 5.5 & 5.6 \\
\hline ts9-5 & 19.9 & 18.6 & 18.4 \\
\hline int10-5 & 17.6 & 16.3 & 16.5 \\
\hline int11-5 & -14.8 & -15.5 & -16.4 \\
\hline
\end{tabular}




\begin{tabular}{|c|c|c|c|}
\hline int1-6 & 0.0 & 0.0 & 0.0 \\
\hline int3-6 & 21.8 & 20.6 & 22.6 \\
\hline int4-6 & -2.5 & -1.6 & -1.1 \\
\hline ts5-6 & 9.9 & 8.2 & 9.9 \\
\hline int6-6 & 3.6 & 2.7 & 4.1 \\
\hline ts7-6 & 13.6 & 11.3 & 12.4 \\
\hline int8-6 & 1.1 & 0.1 & 2.4 \\
\hline ts9-6 & 13.6 & 12.7 & 13.7 \\
\hline int10-6 & -2.9 & -3.9 & -3.0 \\
\hline int11-6 & -26.0 & -26.4 & -26.1 \\
\hline
\end{tabular}

Table S3. Thermochemistry data obtained using rigid rotor approximation for catalysts 3, 4, 5, 6. Enthalpy (2) and Entropy (2) data obtained using Grimme's quasi-rrho approximation; Enthalpy (3) and Entropy (3) data obtained using Cramer and Truhlar's quasi-harmonic approximation.

\begin{tabular}{|c|c|c|c|c|}
\hline Structure & $\begin{array}{c}\text { Enthalpy (2) } \\
\text { (H) }\end{array}$ & $\begin{array}{c}\text { Entropy (2) } \\
(\text { cal/(mol*K)) }\end{array}$ & $\begin{array}{c}\text { Enthalpy (3) } \\
\text { (H) }\end{array}$ & $\begin{array}{c}\text { Entropy (3) } \\
(\text { cal/(mol*K)) }\end{array}$ \\
\hline int1-3 & 0.041 & 275.7 & 0.041 & 266.3 \\
\hline int3-3 & 0.028 & 204.1 & 0.028 & 198.4 \\
\hline int4-3 & 0.031 & 215.6 & 0.031 & 212.1 \\
\hline ts5-3 & 0.031 & 213.0 & 0.031 & 209.7 \\
\hline int6-3 & 0.031 & 218.3 & 0.031 & 214.6 \\
\hline ts 7-3 & 0.031 & 218.6 & 0.031 & 214.4 \\
\hline int8-3 & 0.028 & 199.3 & 0.028 & 194.9 \\
\hline ts9-3 & 0.027 & 198.3 & 0.027 & 194.6 \\
\hline int10-3 & 0.028 & 198.7 & 0.028 & 195.3 \\
\hline int11-3 & 0.040 & 266.8 & 0.040 & 261.7 \\
\hline int1-4 & 0.041 & 272.6 & 0.041 & 265.6 \\
\hline int3-4 & 0.028 & 204.2 & 0.028 & 199.3 \\
\hline int4-4 & 0.031 & 214.6 & 0.031 & 211.6 \\
\hline ts5-4 & 0.031 & 212.9 & 0.031 & 210.0 \\
\hline int6-4 & 0.031 & 217.7 & 0.031 & 214.4 \\
\hline ts 7-4 & 0.031 & 217.8 & 0.031 & 214.3 \\
\hline int8-4 & 0.028 & 199.0 & 0.028 & 195.4 \\
\hline ts9-4 & 0.027 & 196.9 & 0.027 & 193.6 \\
\hline int10-4 & 0.028 & 198.1 & 0.028 & 195.0 \\
\hline int11-4 & 0.041 & 266.5 & 0.041 & 260.1 \\
\hline int1-5 & 0.043 & 282.6 & 0.043 & 275.6 \\
\hline int3-5 & 0.031 & 217.9 & 0.031 & 210.7 \\
\hline int4-5 & 0.033 & 226.8 & 0.033 & 222.1 \\
\hline ts5-5 & 0.033 & 224.6 & 0.033 & 219.9 \\
\hline int6-5 & 0.033 & 230.0 & 0.033 & 224.1 \\
\hline ts 7-5 & 0.033 & 230.1 & 0.033 & 223.9 \\
\hline int8-5 & 0.030 & 212.4 & 0.030 & 207.2 \\
\hline ts9-5 & 0.030 & 209.1 & 0.030 & 204.9 \\
\hline int10-5 & 0.030 & 211.6 & 0.030 & 206.1 \\
\hline int11-5 & 0.043 & 276.2 & 0.043 & 272.1 \\
\hline int1-6 & 0.049 & 308.3 & 0.049 & 304.1 \\
\hline int3-6 & 0.037 & 250.9 & 0.037 & 242.4 \\
\hline int4-6 & 0.039 & 255.3 & 0.039 & 251.7 \\
\hline ts5-6 & 0.039 & 259.8 & 0.039 & 252.2 \\
\hline int6-6 & 0.039 & 258.6 & 0.039 & 252.0 \\
\hline ts7-6 & 0.039 & 258.1 & 0.039 & 252.8 \\
\hline int8-6 & 0.036 & 250.9 & 0.036 & 241.5 \\
\hline ts9-6 & 0.035 & 240.5 & 0.035 & 235.4 \\
\hline int10-6 & 0.035 & 240.6 & 0.035 & 235.9 \\
\hline int11-6 & 0.049 & 308.3 & 0.049 & 304.1 \\
\hline ethylene & 0.004 & 52.3 & 0.004 & 52.3 \\
\hline
\end{tabular}




\begin{tabular}{|c|c|c|c|c|}
\hline ethane & 0.004 & 57.9 & 0.004 & 57.9 \\
\hline phosphine & 0.014 & 124.7 & 0.014 & 122.4 \\
\hline
\end{tabular}

Table S4. Total energy values (E) and Gibbs free energy values (G; as defined in the manuscript) for catalyst (1) and its complexes.

\begin{tabular}{|c|c|c|c|c|c|c|c|c|c|}
\hline structure & $\begin{array}{l}\text { M06 E. } \\
(\text { H) }(E)\end{array}$ & $\begin{array}{c}\text { DLPNO- } \\
\text { CCSD(T) } \\
\text { E (H) }\end{array}$ & $\begin{array}{c}\text { Solvatation } \\
\mathrm{E} \text { in } \\
\text { toluene }\end{array}$ & $\begin{array}{c}\text { Zero point } \\
\text { energy } \\
\text { (kcal/mol) }\end{array}$ & $\begin{array}{c}\text { Entropy } \\
\left(\mathrm{cal} /\left(\mathrm{mol}^{*} \mathbf{K}\right)\right)\end{array}$ & $\begin{array}{c}\text { Thermal } \\
\text { correction } \\
\text { to } \\
\text { enthalpy } \\
\text { (H) }\end{array}$ & $\begin{array}{c}\text { M06 G } \\
\text { (H) }\end{array}$ & $\begin{array}{c}\text { DLPNO- } \\
\text { CCSD(T) } \\
\text { G (H) }\end{array}$ & $\begin{array}{c}\text { D3 } \\
\text { correction } \\
\text { (H) }\end{array}$ \\
\hline int1-1 & -2820.778 & -2813.847 & 0.0020 & 543.3 & 308.4 & 0.055 & -2820.001 & -2813.069 & -0.040 \\
\hline Int3-1 & -1784.805 & -1780.634 & 0.0000 & 371.6 & 232.4 & 0.039 & -1784.284 & -1780.113 & -0.020 \\
\hline int4-1 & -1863.371 & -1858.951 & 0.0023 & 405.3 & 248.8 & 0.042 & -1862.798 & -1858.378 & -0.023 \\
\hline ts5-1 & -1863.359 & -1858.937 & 0.0021 & 404.9 & 241.6 & 0.042 & -1862.784 & -1858.363 & -0.023 \\
\hline int6-1 & -1863.378 & -1858.949 & 0.0012 & 406.7 & 251.1 & 0.043 & -1862.804 & -1858.376 & -0.023 \\
\hline ts7-1 & -1863.362 & -1858.919 & 0.0013 & 406.0 & 248.9 & 0.042 & -1862.789 & -1858.346 & -0.023 \\
\hline int8-1 & -1783.607 & -1779.414 & -0.0003 & 361.8 & 228.5 & 0.038 & -1783.101 & -1778.908 & -0.019 \\
\hline ts9-1 & -1783.586 & -1779.404 & 0.0013 & 358.6 & 224.9 & 0.037 & -1783.082 & -1778.900 & -0.019 \\
\hline int10-1 & -1783.604 & -1779.438 & 0.0020 & 359.5 & 225.0 & 0.038 & -1783.098 & -1778.932 & -0.019 \\
\hline int11-1 & -2819.586 & -2812.665 & 0.0020 & 531.8 & 304.2 & 0.055 & -2818.826 & -2811.904 & -0.040 \\
\hline
\end{tabular}


Table S5. The F-SAPT and SAPT0 contribution for transition states ts5, ts7 and ts9 for catalysts 3 , $4,5,6$.

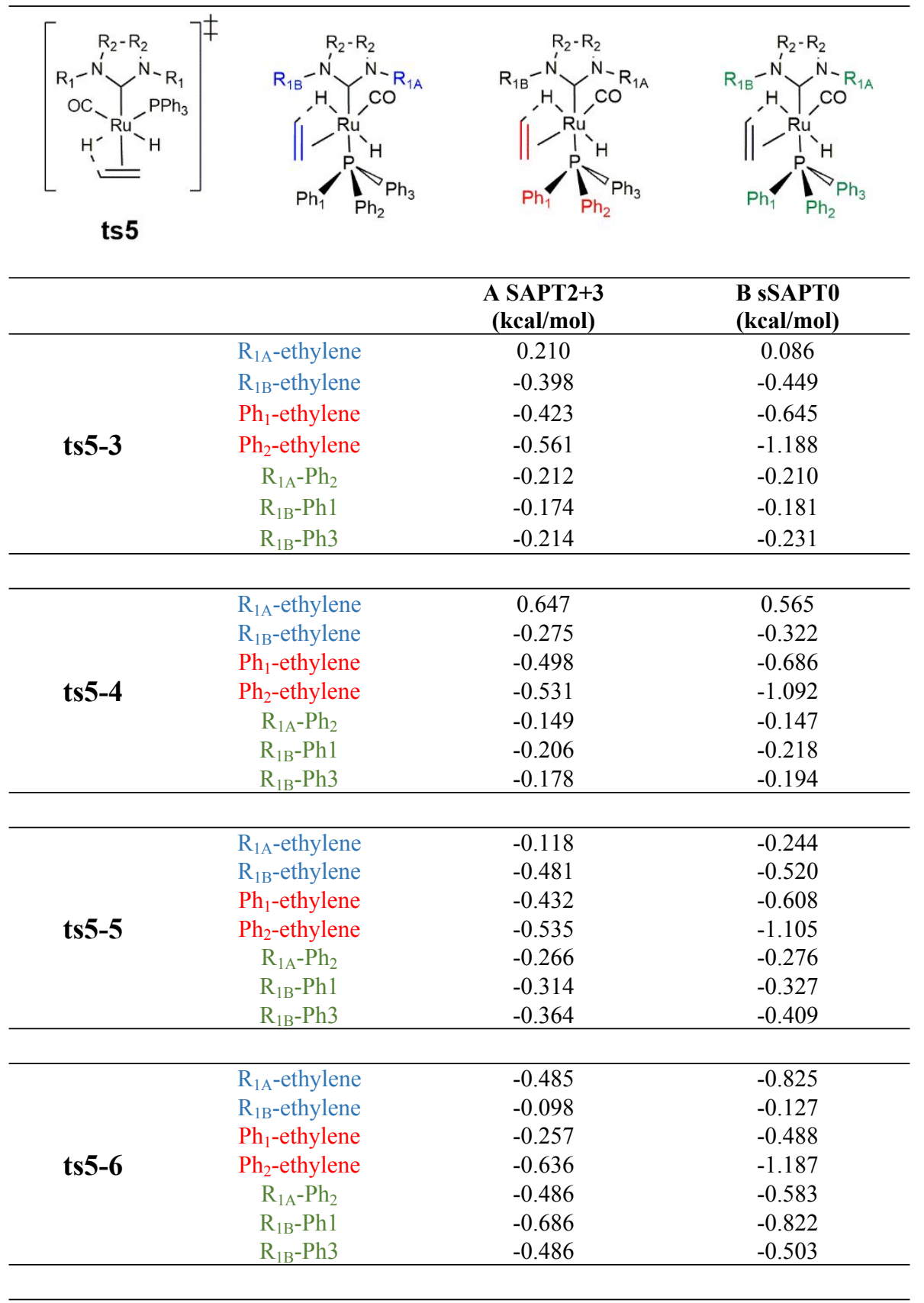

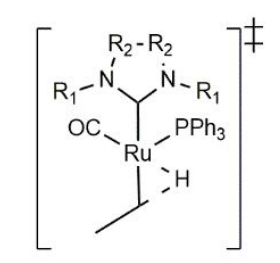

ts7

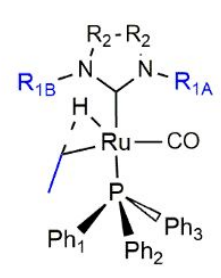

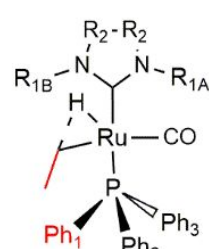

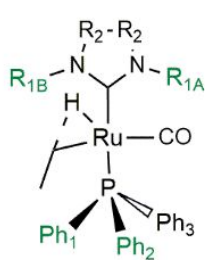

\begin{tabular}{cccc}
\hline & & $\begin{array}{c}\text { A SAPT2+3 } \\
\text { (kcal/mol) }\end{array}$ & $\begin{array}{c}\text { B sSAPT0 } \\
\text { (kcal/mol) }\end{array}$ \\
\hline \multirow{3}{*}{ ts7-3 } & $\mathrm{R}_{1 \mathrm{~A}}$-ethylene & -0.384 & -0.401 \\
& $\mathrm{R}_{1 \mathrm{~B}}-$ ethylene & -0.514 & -0.549 \\
& $\mathrm{Ph}_{1}-\mathrm{ethylene}$ & -0.245 & -0.622 \\
& $\mathrm{R}_{1 \mathrm{~A}}-\mathrm{Ph}_{2}$ & -0.230 & -0.228 \\
& $\mathrm{R}_{1 \mathrm{~B}}-\mathrm{Ph}_{2}$ & -0.522 & -0.562 \\
\hline
\end{tabular}




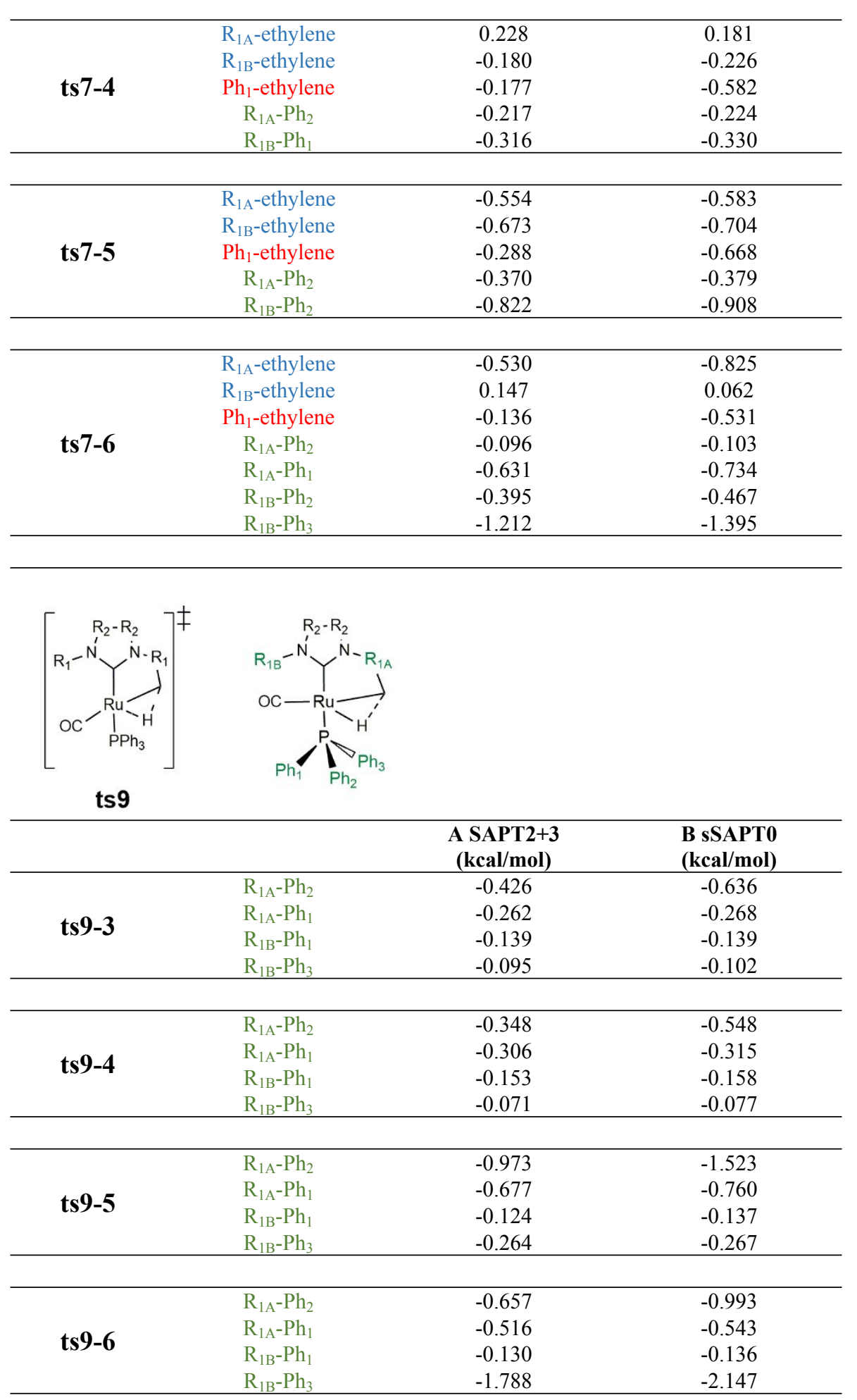


Table S6. The RDG effective density plots for intermediate int3 and transition state ts5, ts7 and ts9 for every considered catalysts $(3,4,5,6)$.

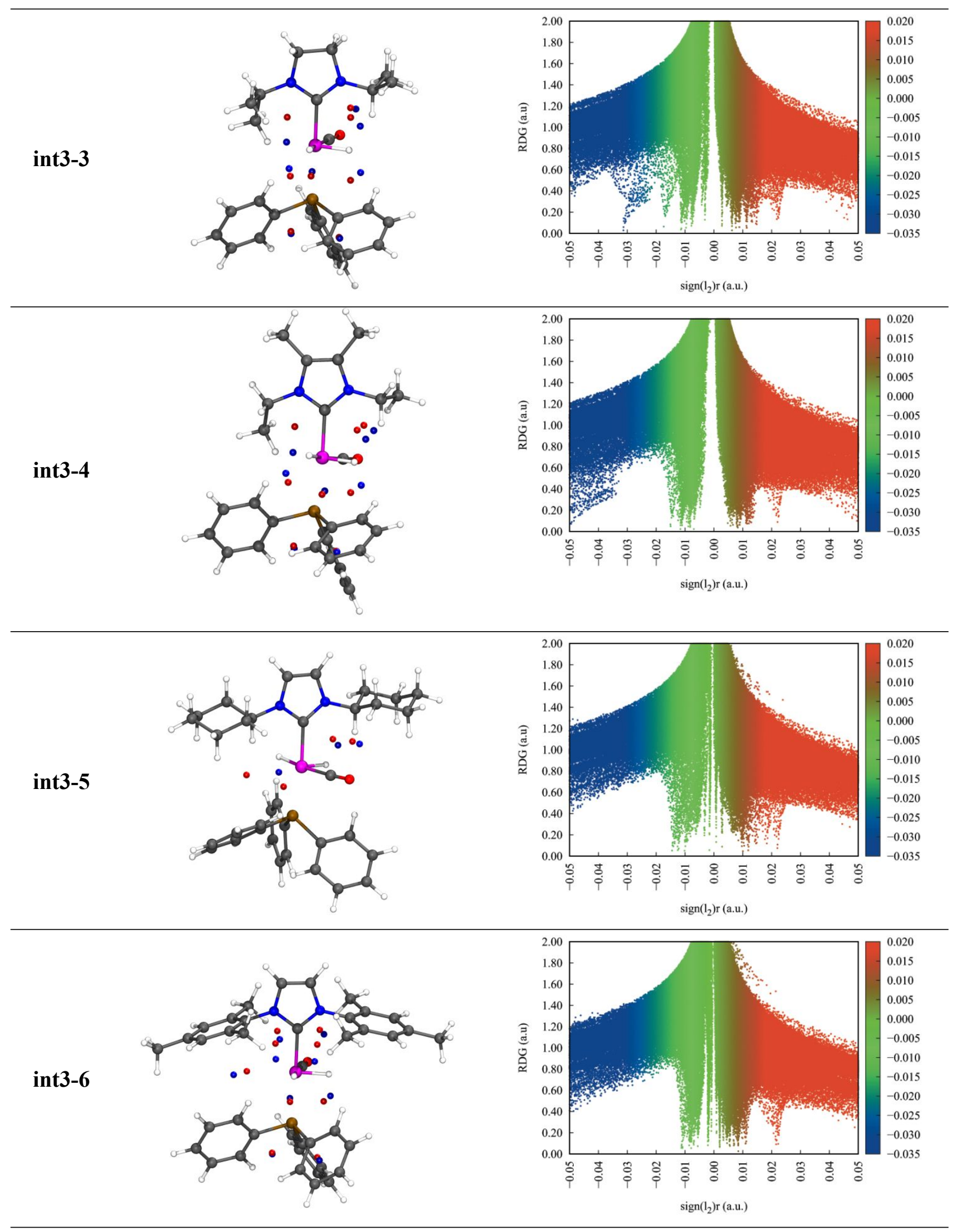




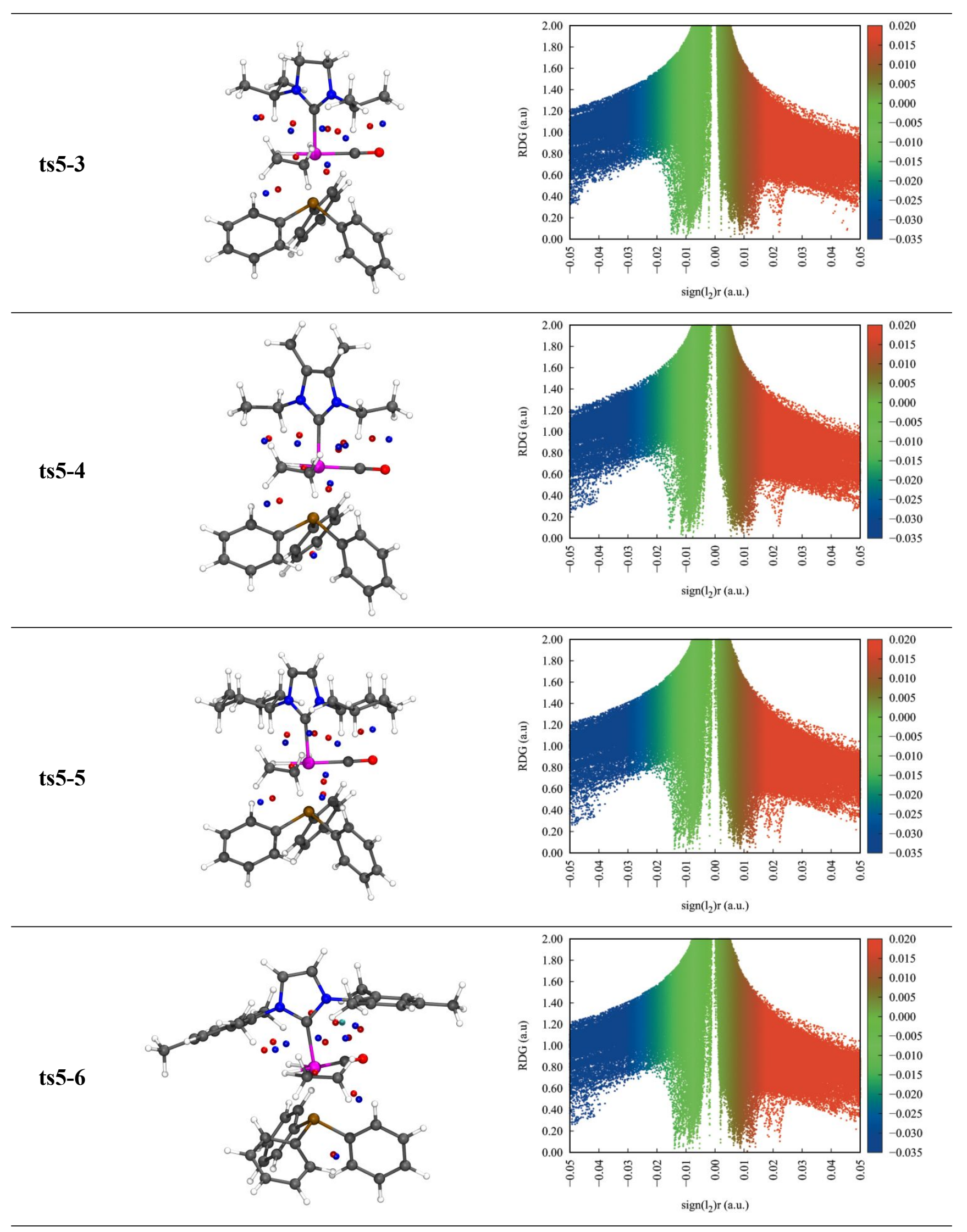




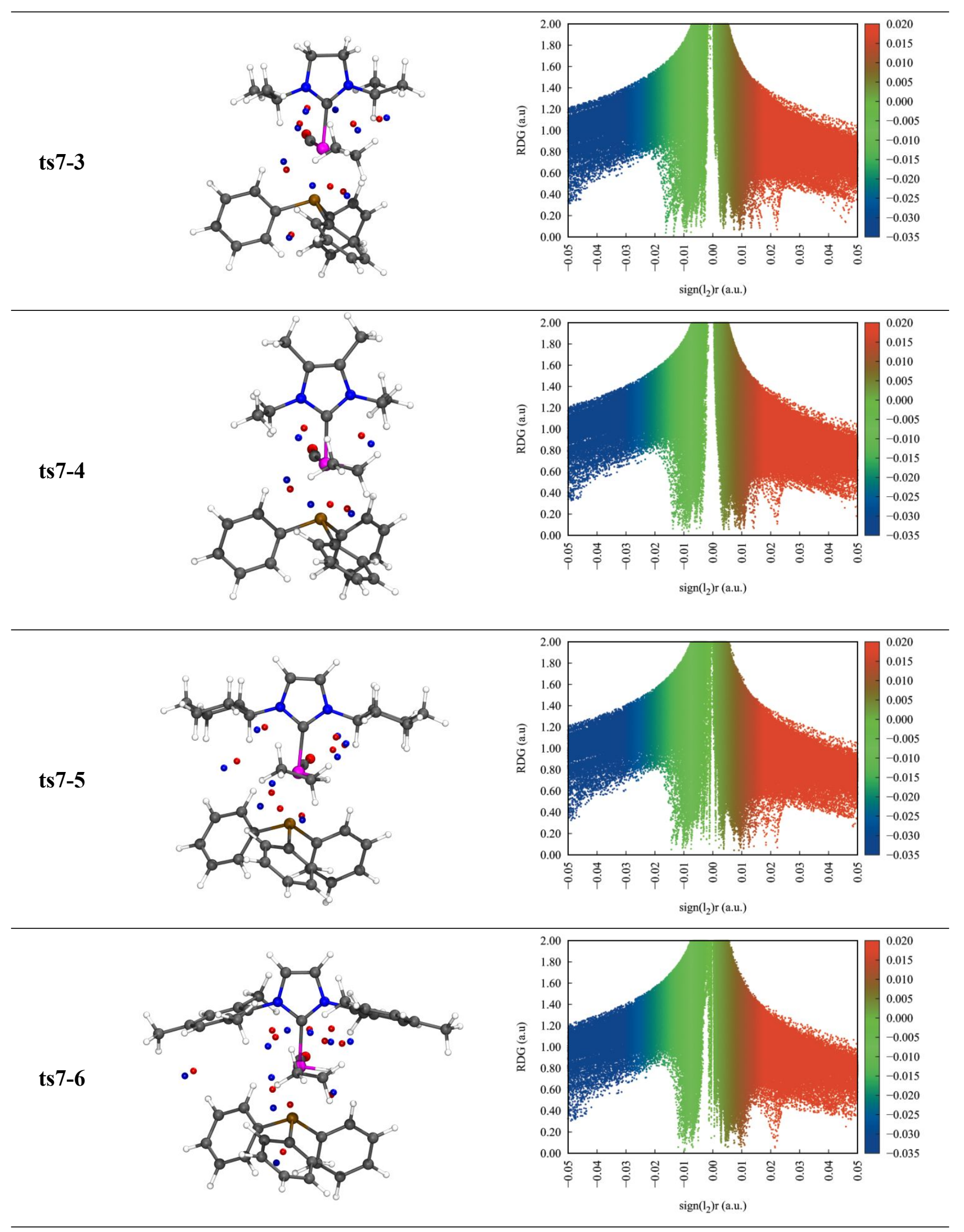




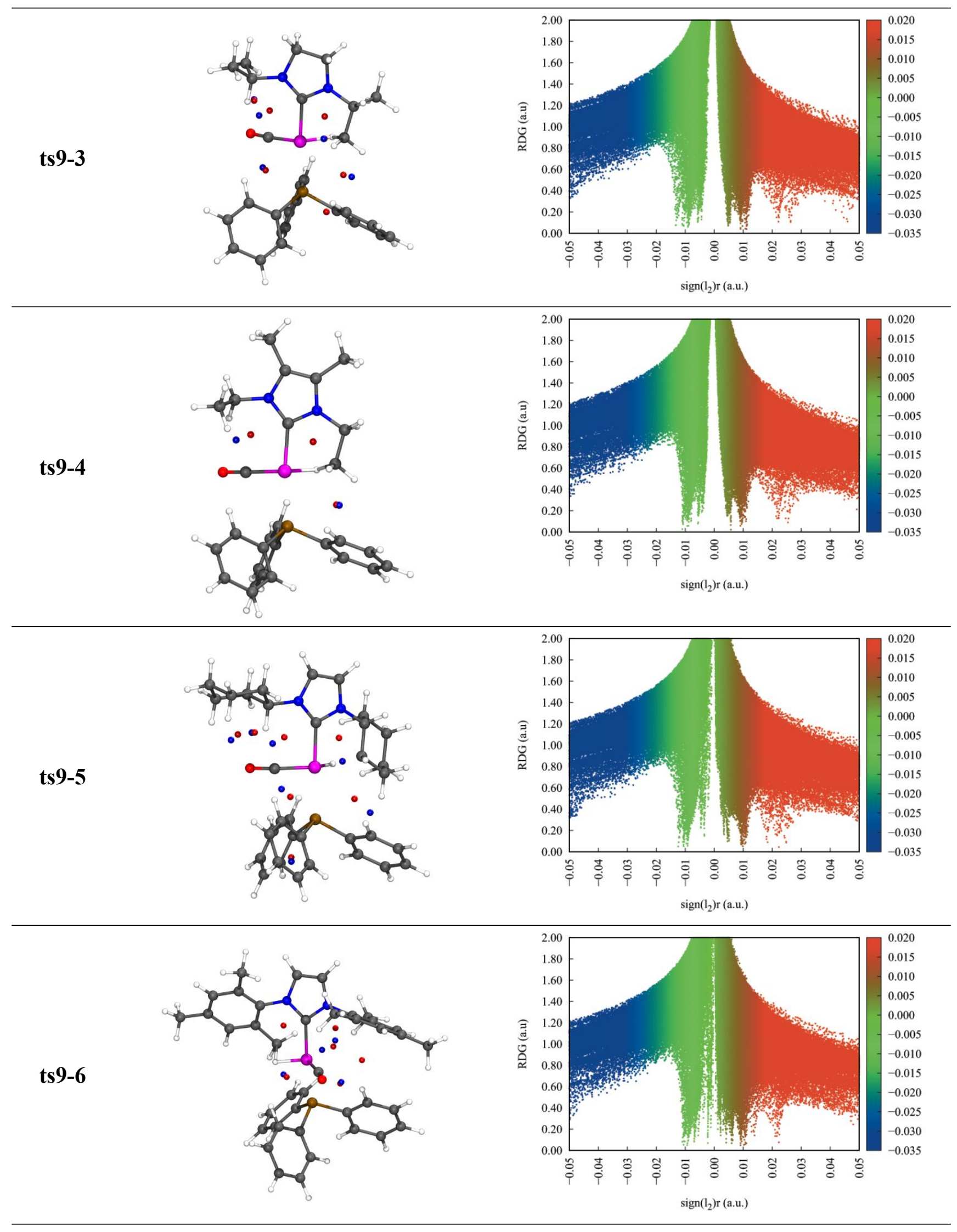


Table S7. The $(3,-1)$ critical points (CPs) and the value of Laplacian of electron density (Laplacian) Reduced Density Gradient $(\boldsymbol{R D} \boldsymbol{G})$, density of all electron, Sign $\left(\lambda_{2}\right)$ r, Potential energy density V(r) and Energy Density for int3, ts5, ts7 and ts9 for all considered catalysts $(3,4,5,6)$.

\begin{tabular}{|c|c|c|c|c|c|c|c|}
\hline \multicolumn{8}{|c|}{ int3 } \\
\hline Catalyst & $\begin{array}{c}\text { CPs } \\
\text { number }\end{array}$ & Laplacian & RDG & $\begin{array}{c}\text { Density of } \\
\text { all } \\
\text { electron }\end{array}$ & Sign $\left(\lambda_{2}\right) r$ & $\begin{array}{c}\text { Potential } \\
\text { energy } \\
\text { density } \\
\text { V(r) }\end{array}$ & $\begin{array}{l}\text { Energy } \\
\text { density }\end{array}$ \\
\hline \multirow{7}{*}{3} & 18 & 4.36E-02 & $4.41 \mathrm{E}-16$ & $1.74 \mathrm{E}-02$ & $-1.74 \mathrm{E}-02$ & $-8.77 \mathrm{E}-03$ & $1.07 \mathrm{E}-03$ \\
\hline & 75 & $1.73 \mathrm{E}-02$ & $7.92 \mathrm{E}-16$ & 7.48E-03 & $-7.48 \mathrm{E}-03$ & $-3.39 \mathrm{E}-03$ & 4.63E-04 \\
\hline & 67 & 0.125485 & $4.90 \mathrm{E}-16$ & $3.14 \mathrm{E}-02$ & $-3.14 \mathrm{E}-02$ & $-3.60 \mathrm{E}-02$ & $-2.30 \mathrm{E}-03$ \\
\hline & 73 & $2.18 \mathrm{E}-02$ & $6.53 \mathrm{E}-16$ & $9.71 \mathrm{E}-03$ & $-9.71 \mathrm{E}-03$ & $-4.49 \mathrm{E}-03$ & $4.86 \mathrm{E}-04$ \\
\hline & 20 & $2.68 \mathrm{E}-02$ & $1.51 \mathrm{E}-15$ & $1.03 \mathrm{E}-02$ & $-1.03 \mathrm{E}-02$ & $-5.26 \mathrm{E}-03$ & 7.19E-04 \\
\hline & 45 & $2.27 \mathrm{E}-02$ & $1.70 \mathrm{E}-15$ & 7.79E-03 & $-7.79 \mathrm{E}-03$ & $-3.80 \mathrm{E}-03$ & $9.33 \mathrm{E}-04$ \\
\hline & 9 & $1.15 \mathrm{E}-02$ & $2.67 \mathrm{E}-15$ & $3.17 \mathrm{E}-03$ & $-3.17 \mathrm{E}-03$ & $-1.80 \mathrm{E}-03$ & $5.45 \mathrm{E}-04$ \\
\hline \multirow{6}{*}{4} & 9 & $1.89 \mathrm{E}-02$ & $2.75 \mathrm{E}-15$ & $5.75 \mathrm{E}-03$ & $5.49 \mathrm{E}-03$ & $-3.38 \mathrm{E}-03$ & $1.14 \mathrm{E}-03$ \\
\hline & 15 & $3.15 \mathrm{E}-02$ & $2.46 \mathrm{E}-16$ & $1.28 \mathrm{E}-02$ & $-1.28 \mathrm{E}-02$ & $-6.22 \mathrm{E}-03$ & $8.28 \mathrm{E}-04$ \\
\hline & 21 & $2.57 \mathrm{E}-02$ & $2.65 \mathrm{E}-16$ & $1.01 \mathrm{E}-02$ & $-1.01 \mathrm{E}-02$ & $-5.05 \mathrm{E}-03$ & $6.90 \mathrm{E}-04$ \\
\hline & 46 & 2.29E-02 & $1.24 \mathrm{E}-15$ & 7.77E-03 & $-7.77 \mathrm{E}-03$ & $-3.82 \mathrm{E}-03$ & $9.58 \mathrm{E}-04$ \\
\hline & 72 & $2.28 \mathrm{E}-02$ & $1.55 \mathrm{E}-16$ & $1.01 \mathrm{E}-02$ & $-1.01 \mathrm{E}-02$ & $-4.69 \mathrm{E}-03$ & $5.02 \mathrm{E}-04$ \\
\hline & 68 & 0.1218271 & $2.96 \mathrm{E}-15$ & $2.88 \mathrm{E}-02$ & $-2.88 \mathrm{E}-02$ & $-3.35 \mathrm{E}-02$ & $-1.52 \mathrm{E}-03$ \\
\hline \multirow{3}{*}{5} & 72 & 3.09E-02 & $1.06 \mathrm{E}-15$ & $1.26 \mathrm{E}-02$ & $-1.26 \mathrm{E}-02$ & $-6.18 \mathrm{E}-03$ & 7.73E-04 \\
\hline & 83 & $1.21 \mathrm{E}-02$ & $1.47 \mathrm{E}-15$ & $3.39 \mathrm{E}-03$ & $-3.39 \mathrm{E}-03$ & $-1.97 \mathrm{E}-03$ & $5.28 \mathrm{E}-04$ \\
\hline & 38 & $3.15 \mathrm{E}-02$ & $2.50 \mathrm{E}-15$ & $1.37 \mathrm{E}-02$ & $-1.37 \mathrm{E}-02$ & $-6.30 \mathrm{E}-03$ & $7.96 \mathrm{E}-04$ \\
\hline \multirow{7}{*}{6} & 25 & $1.75 \mathrm{E}-02$ & $4.06 \mathrm{E}-15$ & 7.91E-03 & $-7.91 \mathrm{E}-03$ & $-3.43 \mathrm{E}-03$ & $4.76 \mathrm{E}-04$ \\
\hline & 40 & $1.76 \mathrm{E}-02$ & 4.30E-15 & 7.92E-03 & $-7.92 \mathrm{E}-03$ & $-3.47 \mathrm{E}-03$ & 4.64E-04 \\
\hline & 63 & $1.79 \mathrm{E}-02$ & $9.19 \mathrm{E}-16$ & $5.61 \mathrm{E}-03$ & $-5.61 \mathrm{E}-03$ & $-3.22 \mathrm{E}-03$ & $6.29 \mathrm{E}-04$ \\
\hline & 79 & $1.84 \mathrm{E}-02$ & $3.20 \mathrm{E}-16$ & $5.77 \mathrm{E}-03$ & $-5.77 \mathrm{E}-03$ & $-2.89 \mathrm{E}-03$ & $8.53 \mathrm{E}-04$ \\
\hline & 73 & $6.24 \mathrm{E}-03$ & $6.57 \mathrm{E}-16$ & $2.13 \mathrm{E}-03$ & $-2.13 \mathrm{E}-03$ & $-9.45 \mathrm{E}-04$ & $3.07 \mathrm{E}-04$ \\
\hline & 31 & $2.17 \mathrm{E}-02$ & $3.48 \mathrm{E}-15$ & 8.67E-03 & $-8.67 \mathrm{E}-03$ & $-4.14 \mathrm{E}-03$ & $6.48 \mathrm{E}-04$ \\
\hline & 84 & $2.28 \mathrm{E}-02$ & $3.02 \mathrm{E}-15$ & 7.07E-03 & $-7.07 \mathrm{E}-03$ & $-3.59 \mathrm{E}-03$ & $1.05 \mathrm{E}-03$ \\
\hline \multicolumn{8}{|c|}{ ts5 } \\
\hline \multirow{7}{*}{3} & 78 & $2.24 \mathrm{E}-02$ & $4.66 \mathrm{E}-16$ & $6.62 \mathrm{E}-03$ & $-6.62 \mathrm{E}-03$ & $-4.03 \mathrm{E}-03$ & $7.90 \mathrm{E}-04$ \\
\hline & 85 & $1.70 \mathrm{E}-02$ & $2.94 \mathrm{E}-15$ & $5.68 \mathrm{E}-03$ & $-5.68 \mathrm{E}-03$ & $-3.05 \mathrm{E}-03$ & $6.01 \mathrm{E}-04$ \\
\hline & 72 & $3.43 \mathrm{E}-02$ & $1.09 \mathrm{E}-15$ & $1.32 \mathrm{E}-02$ & $-1.32 \mathrm{E}-02$ & $-6.34 \mathrm{E}-03$ & $1.11 \mathrm{E}-03$ \\
\hline & 31 & $7.71 \mathrm{E}-04$ & $1.21 \mathrm{E}-15$ & 2.17E-03 & $-2.17 \mathrm{E}-03$ & $-1.10 \mathrm{E}-04$ & $4.14 \mathrm{E}+02$ \\
\hline & 26 & $3.64 \mathrm{E}-02$ & $9.40 \mathrm{E}-16$ & $1.51 \mathrm{E}-02$ & $-1.51 \mathrm{E}-02$ & $-7.34 \mathrm{E}-03$ & 8.77E-04 \\
\hline & 34 & $2.31 \mathrm{E}-02$ & $4.95 \mathrm{E}-16$ & 8.89E-03 & $-8.89 \mathrm{E}-03$ & $-4.43 \mathrm{E}-03$ & $6.71 \mathrm{E}-04$ \\
\hline & 41 & $2.24 \mathrm{E}-02$ & $1.90 \mathrm{E}-16$ & $7.66 \mathrm{E}-03$ & $-7.66 \mathrm{E}-03$ & $-3.70 \mathrm{E}-03$ & $9.53 \mathrm{E}-04$ \\
\hline \multirow{7}{*}{4} & 85 & 2.89E-02 & $6.54 \mathrm{E}-16$ & $8.62 \mathrm{E}-03$ & $-8.62 \mathrm{E}-03$ & $-5.17 \mathrm{E}-03$ & $1.02 \mathrm{E}-03$ \\
\hline & 77 & 2.99E-02 & $7.89 \mathrm{E}-16$ & $1.17 \mathrm{E}-02$ & $-1.17 \mathrm{E}-02$ & $-6.03 E-03$ & $7.28 \mathrm{E}-04$ \\
\hline & 89 & $3.14 \mathrm{E}-02$ & $7.56 \mathrm{E}-16$ & $1.01 \mathrm{E}-02$ & $-1.01 \mathrm{E}-02$ & $-5.35 \mathrm{E}-03$ & $1.24 \mathrm{E}-03$ \\
\hline & 26 & $3.73 \mathrm{E}-02$ & $8.80 \mathrm{E}-16$ & $1.57 \mathrm{E}-02$ & $-1.57 \mathrm{E}-02$ & $-7.49 \mathrm{E}-03$ & $9.18 \mathrm{E}-04$ \\
\hline & 30 & $1.66 \mathrm{E}-02$ & 8.09E-16 & $5.66 \mathrm{E}-03$ & $-5.66 \mathrm{E}-03$ & $-2.99 \mathrm{E}-03$ & $5.74 \mathrm{E}-04$ \\
\hline & 42 & $2.26 \mathrm{E}-02$ & $9.91 \mathrm{E}-16$ & 7.71E-03 & $-7.71 \mathrm{E}-03$ & $-3.72 \mathrm{E}-03$ & $9.68 \mathrm{E}-04$ \\
\hline & 37 & $2.41 \mathrm{E}-02$ & $5.68 \mathrm{E}-16$ & $8.88 \mathrm{E}-03$ & $-8.88 \mathrm{E}-03$ & $-4.57 \mathrm{E}-03$ & 7.32E-04 \\
\hline \multirow{5}{*}{5} & 92 & $1.57 \mathrm{E}-02$ & $1.65 \mathrm{E}-15$ & $5.34 \mathrm{E}-03$ & $-5.34 \mathrm{E}-03$ & $-2.90 \mathrm{E}-03$ & $5.18 \mathrm{E}-04$ \\
\hline & 80 & 3.34E-02 & $1.00 \mathrm{E}-15$ & $1.26 \mathrm{E}-02$ & $-1.26 \mathrm{E}-02$ & $-6.08 \mathrm{E}-03$ & $1.13 \mathrm{E}-03$ \\
\hline & 36 & $3.49 \mathrm{E}-02$ & $6.11 \mathrm{E}-16$ & $1.40 \mathrm{E}-02$ & $-1.40 \mathrm{E}-02$ & $-6.76 \mathrm{E}-03$ & $9.80 \mathrm{E}-04$ \\
\hline & 87 & $2.06 \mathrm{E}-04$ & $5.10 \mathrm{E}-16$ & $6.20 \mathrm{E}-03$ & $-6.20 \mathrm{E}-03$ & $-3.78 \mathrm{E}-03$ & $6.84 \mathrm{E}-04$ \\
\hline & 83 & 2.29E-02 & $8.68 \mathrm{E}-16$ & 7.76E-03 & $-7.76 \mathrm{E}-03$ & $-3.94 \mathrm{E}-03$ & $8.87 \mathrm{E}-04$ \\
\hline
\end{tabular}




\begin{tabular}{|c|c|c|c|c|c|c|c|}
\hline & 42 & $2.43 \mathrm{E}-02$ & $1.42 \mathrm{E}-15$ & $9.07 \mathrm{E}-03$ & $-9.07 \mathrm{E}-03$ & $-4.62 \mathrm{E}-03$ & 7.25E-04 \\
\hline & 47 & $2.26 \mathrm{E}-02$ & $3.65 \mathrm{E}-15$ & $7.67 \mathrm{E}-03$ & $-7.67 \mathrm{E}-03$ & $-3.71 \mathrm{E}-03$ & $9.67 \mathrm{E}-04$ \\
\hline \multirow{7}{*}{6} & 39 & $2.24 \mathrm{E}-02$ & $5.29 \mathrm{E}-15$ & 7.89E-03 & $-7.89 \mathrm{E}-03$ & $-4.05 \mathrm{E}-03$ & 7.81E-04 \\
\hline & 35 & 2.39E-02 & $9.12 \mathrm{E}-16$ & 9.94E-03 & $-9.94 \mathrm{E}-03$ & $-4.84 \mathrm{E}-04$ & $5.72 \mathrm{E}-04$ \\
\hline & 69 & $1.67 \mathrm{E}-02$ & $6.26 \mathrm{E}-16$ & $5.41 \mathrm{E}-03$ & $-5.41 \mathrm{E}-03$ & $-3.01 \mathrm{E}-03$ & $5.77 \mathrm{E}-04$ \\
\hline & 90 & 2.89E-02 & $2.36 \mathrm{E}-15$ & $8.66 \mathrm{E}-03$ & $-8.66 \mathrm{E}-03$ & $-4.86 \mathrm{E}-03$ & 1.19E-03 \\
\hline & 83 & $2.76 \mathrm{E}-02$ & $1.71 \mathrm{E}-15$ & 7.14E-03 & $-7.14 \mathrm{E}-03$ & $-4.46 \mathrm{E}-03$ & $1.22 \mathrm{E}-03$ \\
\hline & 55 & $2.07 \mathrm{E}-02$ & $4.25 \mathrm{E}-16$ & 7.00E-03 & $-7.00 \mathrm{E}-03$ & $-3.39 \mathrm{E}-03$ & 8.89E-04 \\
\hline & 103 & $2.79 \mathrm{E}-02$ & $1.16 \mathrm{E}-15$ & $8.96 \mathrm{E}-03$ & $-8.96 \mathrm{E}-03$ & $-4.69 \mathrm{E}-03$ & $1.15 \mathrm{E}-03$ \\
\hline \multicolumn{8}{|c|}{ ts7 } \\
\hline \multirow{7}{*}{3} & 72 & $5.04 \mathrm{E}-02$ & $7.11 \mathrm{E}-16$ & $1.62 \mathrm{E}-02$ & $-1.62 \mathrm{E}-02$ & $-1.08 \mathrm{E}-02$ & $8.83 \mathrm{E}-04$ \\
\hline & 15 & $9.96 \mathrm{E}-03$ & $5.64 \mathrm{E}-16$ & $3.21 \mathrm{E}-03$ & $-3.21 \mathrm{E}-03$ & $-1.66 \mathrm{E}-03$ & 4.16E-04 \\
\hline & 30 & $3.34 \mathrm{E}-02$ & $9.29 \mathrm{E}-16$ & $1.35 \mathrm{E}-02$ & $-1.35 \mathrm{E}-02$ & $-6.68 \mathrm{E}-03$ & 8.29E-04 \\
\hline & 42 & $1.04 \mathrm{E}-02$ & $1.55 \mathrm{E}-15$ & $2.84 \mathrm{E}-03$ & $-2.84 \mathrm{E}-03$ & $-1.61 \mathrm{E}-03$ & 4.94E-04 \\
\hline & 64 & $1.74 \mathrm{E}-02$ & $6.91 \mathrm{E}-16$ & $5.80 \mathrm{E}-03$ & $-5.80 \mathrm{E}-03$ & $-2.90 \mathrm{E}-03$ & $7.28 \mathrm{E}-04$ \\
\hline & 73 & $2.31 \mathrm{E}-02$ & $2.95 \mathrm{E}-16$ & $9.84 \mathrm{E}-03$ & $-9.84 \mathrm{E}-03$ & $-4.45 \mathrm{E}-03$ & $6.67 \mathrm{E}-04$ \\
\hline & 34 & $2.27 \mathrm{E}-02$ & $2.17 \mathrm{E}-16$ & 7.55E-03 & $-7.55 \mathrm{E}-03$ & $-3.94 \mathrm{E}-03$ & $8.65 \mathrm{E}-04$ \\
\hline \multirow{8}{*}{4} & 20 & $3.09 \mathrm{E}-02$ & $9.61 \mathrm{E}-16$ & $1.20 \mathrm{E}-02$ & $-1.20 \mathrm{E}-02$ & $-6.15 \mathrm{E}-03$ & $7.90 \mathrm{E}-04$ \\
\hline & 69 & $1.82 \mathrm{E}-02$ & $1.71 \mathrm{E}-15$ & $6.14 \mathrm{E}-03$ & $-6.14 \mathrm{E}-03$ & $-3.14 \mathrm{E}-03$ & $7.06 \mathrm{E}-04$ \\
\hline & 31 & $2.42 \mathrm{E}-02$ & $2.59 \mathrm{E}-15$ & $8.10 \mathrm{E}-03$ & $-8.10 \mathrm{E}-03$ & $-4.24 \mathrm{E}-03$ & $9.07 \mathrm{E}-04$ \\
\hline & 59 & $1.70 \mathrm{E}-02$ & $3.96 \mathrm{E}-16$ & $5.58 \mathrm{E}-03$ & $-5.58 \mathrm{E}-03$ & $-2.80 \mathrm{E}-03$ & 7.17E-04 \\
\hline & 71 & $2.44 \mathrm{E}-02$ & $2.81 \mathrm{E}-16$ & $9.98 \mathrm{E}-03$ & $-9.98 \mathrm{E}-03$ & $-4.56 \mathrm{E}-03$ & 7.73E-04 \\
\hline & 88 & $2.00 \mathrm{E}-02$ & $6.01 \mathrm{E}-16$ & $6.33 \mathrm{E}-03$ & $-6.33 E-03$ & $-3.40 \mathrm{E}-03$ & $8.02 \mathrm{E}-04$ \\
\hline & 65 & $3.14 \mathrm{E}-02$ & $2.71 \mathrm{E}-16$ & 8.38E-03 & $-8.38 \mathrm{E}-03$ & $-4.85 E-03$ & $1.49 \mathrm{E}-03$ \\
\hline & 26 & $1.86 \mathrm{E}-02$ & $2.08 \mathrm{E}-15$ & $5.59 \mathrm{E}-03$ & $-5.59 \mathrm{E}-03$ & $-3.10 \mathrm{E}-03$ & 7.71E-04 \\
\hline \multirow{8}{*}{5} & 37 & $3.05 \mathrm{E}-02$ & $2.89 \mathrm{E}-15$ & $1.28 \mathrm{E}-02$ & $-1.28 \mathrm{E}-02$ & $-6.11 \mathrm{E}-03$ & $7.54 \mathrm{E}-04$ \\
\hline & 50 & 8.33E-03 & $1.53 \mathrm{E}-15$ & 2.19E-03 & $-2.19 \mathrm{E}-03$ & $-1.16 \mathrm{E}-03$ & $4.60 \mathrm{E}-04$ \\
\hline & 72 & $1.71 \mathrm{E}-02$ & $4.83 \mathrm{E}-16$ & $5.69 \mathrm{E}-03$ & $-5.69 \mathrm{E}-03$ & $-2.86 \mathrm{E}-03$ & $7.08 \mathrm{E}-04$ \\
\hline & 45 & $2.27 \mathrm{E}-02$ & $1.96 \mathrm{E}-16$ & $7.57 \mathrm{E}-03$ & $-7.57 \mathrm{E}-03$ & $-3.94 \mathrm{E}-03$ & 8.68E-04 \\
\hline & 77 & $2.52 \mathrm{E}-02$ & $5.94 \mathrm{E}-16$ & $1.03 \mathrm{E}-02$ & $-1.03 \mathrm{E}-02$ & $-4.69 \mathrm{E}-03$ & $8.06 \mathrm{E}-04$ \\
\hline & 81 & $1.19 \mathrm{E}-02$ & $1.75 \mathrm{E}-16$ & $4.12 \mathrm{E}-03$ & $-4.12 \mathrm{E}-03$ & $-2.12 \mathrm{E}-03$ & 4.30E-04 \\
\hline & 24 & $9.84 \mathrm{E}-03$ & $1.14 \mathrm{E}-15$ & $3.24 \mathrm{E}-03$ & $-3.24 \mathrm{E}-03$ & $-1.67 \mathrm{E}-03$ & $3.94 \mathrm{E}-04$ \\
\hline & 97 & $4.09 \mathrm{E}-03$ & $2.11 \mathrm{E}-15$ & $1.18 \mathrm{E}-03$ & $-1.18 \mathrm{E}-03$ & $-4.86 \mathrm{E}-04$ & $2.68 \mathrm{E}-04$ \\
\hline \multirow{9}{*}{6} & 85 & $2.75 \mathrm{E}-02$ & $1.77 \mathrm{E}-15$ & $9.13 \mathrm{E}-03$ & $-9.13 \mathrm{E}-03$ & $-4.74 \mathrm{E}-03$ & $1.06 \mathrm{E}-03$ \\
\hline & 56 & $2.02 \mathrm{E}-02$ & $1.76 \mathrm{E}-15$ & $7.41 \mathrm{E}-03$ & $-7.41 \mathrm{E}-03$ & $-3.73 \mathrm{E}-03$ & $6.59 \mathrm{E}-04$ \\
\hline & 84 & $3.25 \mathrm{E}-02$ & $6.81 \mathrm{E}-16$ & $9.86 \mathrm{E}-03$ & $-9.86 \mathrm{E}-03$ & $-5.60 \mathrm{E}-03$ & $1.26 \mathrm{E}-03$ \\
\hline & 25 & $1.93 \mathrm{E}-02$ & $2.03 \mathrm{E}-15$ & $6.94 \mathrm{E}-03$ & $-6.94 \mathrm{E}-03$ & $-3.48 \mathrm{E}-03$ & $6.70 \mathrm{E}-04$ \\
\hline & 52 & $2.84 \mathrm{E}-02$ & $2.92 \mathrm{E}-15$ & 8.29E-03 & $-8.29 \mathrm{E}-03$ & $-4.73 \mathrm{E}-03$ & $1.19 \mathrm{E}-03$ \\
\hline & 120 & $2.27 \mathrm{E}-03$ & $1.79 \mathrm{E}-14$ & $5.46 \mathrm{E}-04$ & $-5.46 \mathrm{E}-04$ & $-2.08 \mathrm{E}-04$ & $1.79 \mathrm{E}-04$ \\
\hline & 36 & $2.31 \mathrm{E}-02$ & $1.72 \mathrm{E}-15$ & $9.76 \mathrm{E}-03$ & $-9.76 \mathrm{E}-03$ & $-4.68 \mathrm{E}-03$ & $5.43 \mathrm{E}-04$ \\
\hline & 75 & $2.03 \mathrm{E}-02$ & $1.74 \mathrm{E}-15$ & $7.21 \mathrm{E}-03$ & $-7.21 \mathrm{E}-03$ & $-3.50 \mathrm{E}-03$ & 7.91E-04 \\
\hline & 86 & $3.35 \mathrm{E}-02$ & $1.44 \mathrm{E}-15$ & $1.20 \mathrm{E}-02$ & $-1.20 \mathrm{E}-02$ & $-5.79 \mathrm{E}-03$ & $1.29 \mathrm{E}-03$ \\
\hline \multicolumn{8}{|c|}{ ts9 } \\
\hline \multirow{4}{*}{3} & 8 & $1.85 \mathrm{E}-02$ & $7.91 \mathrm{E}-16$ & $5.03 \mathrm{E}-03$ & $-5.03 \mathrm{E}-03$ & $-3.08 \mathrm{E}-03$ & 7.72E-04 \\
\hline & 26 & $3.98 \mathrm{E}-02$ & $7.72 \mathrm{E}-16$ & $1.34 \mathrm{E}-02$ & $-1.34 \mathrm{E}-02$ & $-6.98 \mathrm{E}-03$ & $1.49 \mathrm{E}-03$ \\
\hline & 73 & $1.79 \mathrm{E}-02$ & $6.59 \mathrm{E}-16$ & $5.76 \mathrm{E}-03$ & $-5.76 \mathrm{E}-03$ & $-2.84 \mathrm{E}-03$ & $8.20 \mathrm{E}-04$ \\
\hline & 29 & $2.15 \mathrm{E}-02$ & $3.97 \mathrm{E}-16$ & $9.10 \mathrm{E}-03$ & $-9.10 \mathrm{E}-03$ & $-4.30 \mathrm{E}-03$ & $5.34 \mathrm{E}-04$ \\
\hline \multirow{3}{*}{4} & 14 & $3.86 \mathrm{E}-02$ & $1.98 \mathrm{E}-16$ & $1.11 \mathrm{E}-02$ & $-1.11 \mathrm{E}-02$ & $-6.31 \mathrm{E}-03$ & $1.68 \mathrm{E}-03$ \\
\hline & 59 & $1.89 \mathrm{E}-02$ & 4.24E-16 & $5.70 \mathrm{E}-03$ & $-5.70 \mathrm{E}-03$ & $-2.93 \mathrm{E}-03$ & $8.95 \mathrm{E}-04$ \\
\hline & 53 & $-5.16 \mathrm{E}-02$ & $0.1+\mathrm{e} 3$ & 0.1101604 & -0.1101604 & $-9.00 \mathrm{E}-02$ & $-5.14 \mathrm{E}-02$ \\
\hline 5 & 9 & $1.31 \mathrm{E}-02$ & $1.32 \mathrm{E}-15$ & $3.56 \mathrm{E}-03$ & $-3.56 \mathrm{E}-03$ & $-2.18 \mathrm{E}-03$ & $5.45 \mathrm{E}-04$ \\
\hline \multicolumn{8}{|c|}{ S13 } \\
\hline
\end{tabular}




\begin{tabular}{|c|c|c|c|c|c|c|c|}
\hline & 38 & $3.78 \mathrm{E}-02$ & $3.53 \mathrm{E}-16$ & $1.25 \mathrm{E}-02$ & $-1.25 \mathrm{E}-02$ & $-6.52 \mathrm{E}-03$ & $1.47 \mathrm{E}-03$ \\
\hline & 20 & $1.70 \mathrm{E}-02$ & $5.41 \mathrm{E}-16$ & 4.67E-03 & $-4.67 \mathrm{E}-03$ & $-2.89 \mathrm{E}-03$ & $6.74 \mathrm{E}-04$ \\
\hline & 39 & $2.57 \mathrm{E}-02$ & $9.25 \mathrm{E}-16$ & $1.07 \mathrm{E}-02$ & $-1.07 \mathrm{E}-02$ & $-4.88 \mathrm{E}-03$ & 7.77E-04 \\
\hline & 82 & $2.69 \mathrm{E}-02$ & $2.22 \mathrm{E}-15$ & 0.2420516 & $-9.63 \mathrm{E}-03$ & $-4.72 \mathrm{E}-03$ & $1.01 \mathrm{E}-03$ \\
\hline & 42 & $1.65 \mathrm{E}-02$ & 3.33E-15 & $6.92 \mathrm{E}-03$ & $-6.92 \mathrm{E}-03$ & $-3.58 \mathrm{E}-03$ & 2.66E-04 \\
\hline 6 & 24 & $1.90 \mathrm{E}-02$ & $2.38 \mathrm{E}-15$ & $5.61 \mathrm{E}-03$ & $5.61 \mathrm{E}-03$ & $-3.41 \mathrm{E}-03$ & $6.74 \mathrm{E}-04$ \\
\hline o & 87 & $2.05 \mathrm{E}-02$ & $1.47 \mathrm{E}-15$ & 7.03E-03 & $-7.03 \mathrm{E}-03$ & $-3.38 \mathrm{E}-03$ & 8.74E-04 \\
\hline & 36 & $2.11 \mathrm{E}-02$ & $1.38 \mathrm{E}-15$ & $9.09 \mathrm{E}-03$ & $-9.09 \mathrm{E}-03$ & $-4.26 \mathrm{E}-03$ & $5.06 \mathrm{E}-04$ \\
\hline
\end{tabular}

Table S8. The $(3,+1)$ critical points $(\mathrm{CPs})$ and the value of Laplacian of electron density (Laplacian) Reduced Density Gradient $(\boldsymbol{R D} \boldsymbol{D})$, density of all electron, Sign $\left(\lambda_{2}\right)$ r, Potential energy density $V(r)$ and Energy Density for int3, ts5, ts7 and ts9 for all considered catalysts $(3,4,5,6)$.

\begin{tabular}{|c|c|c|c|c|c|c|c|}
\hline \multicolumn{8}{|c|}{ int3 } \\
\hline catalyst & $\begin{array}{c}\text { CPs } \\
\text { number }\end{array}$ & Laplacian & RDG & $\begin{array}{l}\text { Density of } \\
\text { all } \\
\text { electron }\end{array}$ & $\operatorname{Sign}\left(\lambda_{2}\right) r$ & $\begin{array}{c}\text { Potential } \\
\text { energy } \\
\text { density } \\
\text { V(r) }\end{array}$ & $\begin{array}{l}\text { Energy } \\
\text { density }\end{array}$ \\
\hline \multirow{7}{*}{3} & 27 & $3.10 \mathrm{E}-02$ & $1.52 \mathrm{E}-16$ & 8.73E-03 & 8.73E-03 & $-5.19 \mathrm{E}-03$ & $1.28 \mathrm{E}-03$ \\
\hline & 46 & $2.16 \mathrm{E}-02$ & $5.06 \mathrm{E}-16$ & $7.58 \mathrm{E}-03$ & $7.58 \mathrm{E}-03$ & $-3.87 \mathrm{E}-03$ & 7.64E-04 \\
\hline & 70 & $2.66 \mathrm{E}-02$ & $8.18 \mathrm{E}-16$ & 8.84E-03 & 8.84E-03 & $-4.88 \mathrm{E}-03$ & 8.92E-04 \\
\hline & 71 & $2.06 \mathrm{E}-02$ & $1.77 \mathrm{E}-15$ & $6.26 \mathrm{E}-03$ & $6.26 \mathrm{E}-03$ & $-3.71 E-03$ & $7.22 \mathrm{E}-04$ \\
\hline & 65 & $6.54 \mathrm{E}-02$ & $1.03 \mathrm{E}-15$ & $1.47 \mathrm{E}-02$ & $1.47 \mathrm{E}-02$ & $-1.11 \mathrm{E}-02$ & $2.64 \mathrm{E}-03$ \\
\hline & 24 & 4.72E-02 & $8.24 \mathrm{E}-16$ & $1.31 \mathrm{E}-02$ & $1.31 \mathrm{E}-02$ & $-8.35 \mathrm{E}-03$ & $1.72 \mathrm{E}-03$ \\
\hline & 12 & $1.06 \mathrm{E}-02$ & $2.14 \mathrm{E}-15$ & $2.79 \mathrm{E}-03$ & $2.79 \mathrm{E}-03$ & $-1.46 \mathrm{E}-03$ & $5.96 \mathrm{E}-04$ \\
\hline \multirow{6}{*}{4} & 13 & $1.38 \mathrm{E}-02$ & $5.56 \mathrm{E}-16$ & $4.01 \mathrm{E}-03$ & $4.01 \mathrm{E}-03$ & $-2.20 \mathrm{E}-03$ & $6.22 \mathrm{E}-04$ \\
\hline & 23 & $3.67 \mathrm{E}-02$ & $6.62 \mathrm{E}-16$ & $1.10 \mathrm{E}-02$ & $1.10 \mathrm{E}-02$ & $-6.46 \mathrm{E}-03$ & $1.35 \mathrm{E}-03$ \\
\hline & 66 & $6.18 \mathrm{E}-02$ & $7.82 \mathrm{E}-16$ & $1.42 \mathrm{E}-02$ & $1.42 \mathrm{E}-02$ & $-1.04 \mathrm{E}-02$ & $2.54 \mathrm{E}-03$ \\
\hline & 28 & $2.95 \mathrm{E}-02$ & $6.86 \mathrm{E}-16$ & 8.57E-03 & 8.57E-03 & $-4.98 \mathrm{E}-03$ & $1.20 \mathrm{E}-03$ \\
\hline & 71 & 2.79E-02 & $1.12 \mathrm{E}-15$ & $9.07 \mathrm{E}-03$ & $9.07 \mathrm{E}-03$ & $-5.13 E-03$ & $9.37 \mathrm{E}-04$ \\
\hline & 47 & $2.16 \mathrm{E}-02$ & $6.54 \mathrm{E}-15$ & 7.57E-03 & $7.57 \mathrm{E}-03$ & $-3.86 \mathrm{E}-03$ & $7.67 \mathrm{E}-04$ \\
\hline \multirow{4}{*}{5} & 41 & $3.32 \mathrm{E}-02$ & $1.06 \mathrm{E}-15$ & $9.59 \mathrm{E}-03$ & $9.59 \mathrm{E}-03$ & $-5.75 E-03$ & $1.28 \mathrm{E}-03$ \\
\hline & 36 & $2.46 \mathrm{E}-03$ & $3.86 \mathrm{E}-15$ & 7.79E-04 & 7.79E-04 & $-2.70 \mathrm{E}-04$ & $1.72 \mathrm{E}-04$ \\
\hline & 81 & $1.16 \mathrm{E}-02$ & $3.47 \mathrm{E}-15$ & $2.84 \mathrm{E}-03$ & $2.84 \mathrm{E}-03$ & $-1.59 \mathrm{E}-03$ & $6.58 \mathrm{E}-04$ \\
\hline & 71 & $3.68 \mathrm{E}-02$ & $1.89 \mathrm{E}-15$ & $1.19 \mathrm{E}-02$ & $1.19 \mathrm{E}-02$ & $-6.87 \mathrm{E}-03$ & $1.16 \mathrm{E}-03$ \\
\hline \multirow{7}{*}{6} & 35 & $2.48 \mathrm{E}-02$ & 7.87E-16 & 8.34E-03 & $8.34 \mathrm{E}-03$ & $-4.41 E-03$ & 8.94E-04 \\
\hline & 78 & $2.14 \mathrm{E}-02$ & $1.16 \mathrm{E}-15$ & $6.94 \mathrm{E}-03$ & $6.94 \mathrm{E}-03$ & $-3.58 \mathrm{E}-03$ & $8.86 \mathrm{E}-04$ \\
\hline & 62 & $6.64 \mathrm{E}-03$ & $5.38 \mathrm{E}-16$ & $2.01 \mathrm{E}-03$ & $2.01 \mathrm{E}-03$ & $-9.68 \mathrm{E}-04$ & $3.47 \mathrm{E}-04$ \\
\hline & 28 & $1.84 \mathrm{E}-02$ & $2.54 \mathrm{E}-15$ & $5.54 \mathrm{E}-03$ & $5.54 \mathrm{E}-03$ & $-3.07 \mathrm{E}-03$ & $7.69 \mathrm{E}-04$ \\
\hline & 58 & $1.08 \mathrm{E}-02$ & $6.13 \mathrm{E}-16$ & $3.27 \mathrm{E}-03$ & $3.27 \mathrm{E}-03$ & $-1.74 \mathrm{E}-03$ & 4.77E-04 \\
\hline & 46 & $1.79 \mathrm{E}-02$ & $3.33 \mathrm{E}-15$ & $5.41 \mathrm{E}-03$ & $5.41 \mathrm{E}-03$ & $-3.07 \mathrm{E}-03$ & $7.03 \mathrm{E}-04$ \\
\hline & 74 & $1.44 \mathrm{E}-02$ & $1.45 \mathrm{E}-15$ & $4.56 \mathrm{E}-03$ & $4.56 \mathrm{E}-03$ & $-2.55 \mathrm{E}-03$ & $5.28 \mathrm{E}-04$ \\
\hline \multicolumn{8}{|c|}{ ts5 } \\
\hline \multirow{8}{*}{3} & 77 & $2.12 \mathrm{E}-02$ & $4.58 \mathrm{E}-16$ & $4.85 \mathrm{E}-03$ & $4.85 \mathrm{E}-03$ & $-3.00 \mathrm{E}-03$ & $1.14 \mathrm{E}-03$ \\
\hline & 70 & $3.97 \mathrm{E}-02$ & $6.75 \mathrm{E}-15$ & $1.26 \mathrm{E}-02$ & $1.26 \mathrm{E}-02$ & $-7.61 \mathrm{E}-03$ & $1.16 \mathrm{E}-03$ \\
\hline & 81 & $1.95 \mathrm{E}-02$ & $4.99 \mathrm{E}-16$ & $5.36 \mathrm{E}-03$ & $5.36 \mathrm{E}-03$ & $-3.18 \mathrm{E}-03$ & $8.52 \mathrm{E}-04$ \\
\hline & 30 & 4.76E-02 & 7.32E-16 & $1.33 \mathrm{E}-02$ & $1.33 \mathrm{E}-02$ & $-8.61 \mathrm{E}-03$ & $1.65 \mathrm{E}-03$ \\
\hline & 32 & $8.18 \mathrm{E}-03$ & $2.25 \mathrm{E}-15$ & $2.13 \mathrm{E}-03$ & 2.13E-03 & $-1.14 \mathrm{E}-03$ & $4.51 \mathrm{E}-04$ \\
\hline & 36 & $2.70 \mathrm{E}-02$ & $1.28 \mathrm{E}-15$ & $8.56 \mathrm{E}-03$ & $8.56 \mathrm{E}-03$ & $-4.80 \mathrm{E}-03$ & $9.76 \mathrm{E}-04$ \\
\hline & 45 & $2.47 \mathrm{E}-02$ & $7.73 \mathrm{E}-16$ & $6.74 \mathrm{E}-03$ & $6.74 \mathrm{E}-03$ & $-3.97 \mathrm{E}-03$ & $1.11 \mathrm{E}-03$ \\
\hline & 57 & 0.1938043 & 100 & $6.49 \mathrm{E}-02$ & $6.49 \mathrm{E}-02$ & $-7.62 \mathrm{E}-02$ & $-1.39 \mathrm{E}-02$ \\
\hline
\end{tabular}




\begin{tabular}{|c|c|c|c|c|c|c|c|}
\hline \multirow{8}{*}{4} & 34 & $4.50 \mathrm{E}-02$ & $6.57 \mathrm{E}-16$ & $1.26 \mathrm{E}-02$ & $1.26 \mathrm{E}-02$ & $-7.97 \mathrm{E}-03$ & $1.64 \mathrm{E}-03$ \\
\hline & 29 & $1.90 \mathrm{E}-02$ & $5.35 \mathrm{E}-16$ & $5.49 \mathrm{E}-03$ & $5.49 \mathrm{E}-03$ & $-3.09 \mathrm{E}-03$ & $8.25 \mathrm{E}-04$ \\
\hline & 39 & 2.79E-02 & $2.52 \mathrm{E}-16$ & $8.66 \mathrm{E}-03$ & $8.66 \mathrm{E}-03$ & $-4.92 \mathrm{E}-03$ & $1.02 \mathrm{E}-03$ \\
\hline & 76 & $3.28 \mathrm{E}-02$ & $2.02 \mathrm{E}-15$ & $1.16 \mathrm{E}-02$ & $1.16 \mathrm{E}-02$ & $-6.47 \mathrm{E}-03$ & 8.59E-04 \\
\hline & 81 & $2.58 \mathrm{E}-02$ & $1.46 \mathrm{E}-15$ & $9.75 \mathrm{E}-03$ & $9.75 \mathrm{E}-03$ & $-4.94 \mathrm{E}-03$ & $7.54 \mathrm{E}-04$ \\
\hline & 82 & 2.29E-02 & $1.09 \mathrm{E}-15$ & $5.24 \mathrm{E}-03$ & $5.24 \mathrm{E}-03$ & $-3.19 \mathrm{E}-03$ & $1.27 \mathrm{E}-03$ \\
\hline & 62 & 0.1944052 & 10 & $6.17 \mathrm{E}-02$ & $6.17 \mathrm{E}-02$ & $-7.19 \mathrm{E}-02$ & $-1.17 \mathrm{E}-02$ \\
\hline & 47 & $2.46 \mathrm{E}-02$ & $1.48 \mathrm{E}-15$ & $6.80 \mathrm{E}-03$ & $6.80 \mathrm{E}-03$ & $-3.99 \mathrm{E}-03$ & $1.08 \mathrm{E}-03$ \\
\hline \multirow{8}{*}{5} & 86 & $2.03 \mathrm{E}-02$ & $9.54 \mathrm{E}-16$ & $4.81 \mathrm{E}-03$ & $4.81 \mathrm{E}-03$ & $-3.01 \mathrm{E}-03$ & $1.03 \mathrm{E}-03$ \\
\hline & 90 & $1.80 \mathrm{E}-02$ & $2.93 \mathrm{E}-16$ & $5.13 \mathrm{E}-03$ & $5.13 \mathrm{E}-03$ & $-3.03 \mathrm{E}-03$ & $7.30 \mathrm{E}-04$ \\
\hline & 76 & $3.60 \mathrm{E}-02$ & $6.11 \mathrm{E}-16$ & $1.19 \mathrm{E}-02$ & $1.19 \mathrm{E}-02$ & $-6.93 \mathrm{E}-03$ & $1.03 \mathrm{E}-03$ \\
\hline & 40 & 4.49E-02 & $6.84 \mathrm{E}-16$ & $1.28 \mathrm{E}-02$ & $1.28 \mathrm{E}-02$ & $-8.10 \mathrm{E}-03$ & $1.56 \mathrm{E}-03$ \\
\hline & 81 & $2.03 \mathrm{E}-02$ & $7.15 \mathrm{E}-16$ & 7.70E-03 & $7.70 \mathrm{E}-03$ & $-3.79 \mathrm{E}-03$ & $6.47 \mathrm{E}-04$ \\
\hline & 52 & $2.45 \mathrm{E}-02$ & $1.58 \mathrm{E}-15$ & $6.75 \mathrm{E}-03$ & $6.75 \mathrm{E}-03$ & $-3.95 \mathrm{E}-03$ & $1.08 \mathrm{E}-03$ \\
\hline & 65 & $1.92 \mathrm{E}-01$ & 100 & $6.18 \mathrm{E}-02$ & $6.18 \mathrm{E}-02$ & $-7.16 \mathrm{E}-02$ & $-1.19 \mathrm{E}-02$ \\
\hline & 44 & $2.85 \mathrm{E}-02$ & $1.62 \mathrm{E}-15$ & $8.70 \mathrm{E}-03$ & $8.70 \mathrm{E}-03$ & $-4.98 \mathrm{E}-03$ & $1.08 \mathrm{E}-03$ \\
\hline \multirow{10}{*}{6} & 29 & $1.34 \mathrm{E}-02$ & $3.48 \mathrm{E}-15$ & $3.83 \mathrm{E}-03$ & $3.83 \mathrm{E}-03$ & $-2.08 \mathrm{E}-03$ & $6.40 \mathrm{E}-04$ \\
\hline & 27 & $2.49 \mathrm{E}-02$ & $2.27 \mathrm{E}-15$ & 7.29E-03 & 7.29E-03 & $-4.14 \mathrm{E}-03$ & $1.04 \mathrm{E}-03$ \\
\hline & 75 & $1.96 \mathrm{E}-02$ & $1.11 \mathrm{E}-15$ & $5.10 \mathrm{E}-03$ & $5.10 \mathrm{E}-03$ & $-3.19 \mathrm{E}-03$ & $8.61 \mathrm{E}-04$ \\
\hline & 88 & $1.42 \mathrm{E}-02$ & $1.64 \mathrm{E}-15$ & 4.77E-03 & 4.77E-03 & $-2.44 \mathrm{E}-03$ & $5.52 \mathrm{E}-04$ \\
\hline & 92 & $2.10 \mathrm{E}-02$ & $8.09 \mathrm{E}-16$ & $5.66 \mathrm{E}-03$ & $5.66 \mathrm{E}-03$ & $-3.02 \mathrm{E}-03$ & $1.12 \mathrm{E}-03$ \\
\hline & 99 & $2.10 \mathrm{E}-02$ & $2.09 \mathrm{E}-15$ & $8.49 \mathrm{E}-03$ & $8.49 \mathrm{E}-03$ & $-4.19 \mathrm{E}-03$ & $5.30 \mathrm{E}-04$ \\
\hline & 68 & 0.1904498 & 100 & $6.20 \mathrm{E}-02$ & $6.20 \mathrm{E}-02$ & $-7.21 \mathrm{E}-02$ & $-1.23 \mathrm{E}-02$ \\
\hline & 71 & $1.60 \mathrm{E}-02$ & $1.20 \mathrm{E}-15$ & $4.66 \mathrm{E}-03$ & $4.66 \mathrm{E}-03$ & $-2.66 \mathrm{E}-03$ & $6.64 \mathrm{E}-04$ \\
\hline & 61 & $2.30 \mathrm{E}-02$ & $1.72 \mathrm{E}-15$ & $6.36 \mathrm{E}-03$ & $6.36 \mathrm{E}-03$ & $-3.68 \mathrm{E}-03$ & $1.04 \mathrm{E}-03$ \\
\hline & 50 & $6.57 \mathrm{E}-03$ & $8.52 \mathrm{E}-15$ & $1.90 \mathrm{E}-03$ & $1.90 \mathrm{E}-03$ & $-8.97 \mathrm{E}-04$ & $3.72 \mathrm{E}-04$ \\
\hline \multicolumn{8}{|c|}{ ts7 } \\
\hline \multirow{8}{*}{3} & 48 & $1.49 \mathrm{E}-02$ & $4.00 \mathrm{E}-15$ & $3.96 \mathrm{E}-03$ & $3.96 \mathrm{E}-03$ & $-2.27 \mathrm{E}-03$ & $7.31 \mathrm{E}-04$ \\
\hline & 38 & $2.52 \mathrm{E}-02$ & $9.74 \mathrm{E}-16$ & 7.30E-03 & 7.30E-03 & $-4.15 \mathrm{E}-03$ & $1.07 \mathrm{E}-03$ \\
\hline & 70 & $2.59 \mathrm{E}-02$ & $5.58 \mathrm{E}-16$ & $9.32 \mathrm{E}-03$ & $9.32 \mathrm{E}-03$ & $-5.02 \mathrm{E}-03$ & $7.25 \mathrm{E}-04$ \\
\hline & 40 & $9.54 \mathrm{E}-03$ & $1.71 \mathrm{E}-15$ & $2.49 \mathrm{E}-03$ & $2.49 \mathrm{E}-03$ & $-1.31 \mathrm{E}-03$ & $5.38 \mathrm{E}-04$ \\
\hline & 35 & $3.93 \mathrm{E}-02$ & $4.41 \mathrm{E}-16$ & $1.33 \mathrm{E}-02$ & $1.33 \mathrm{E}-02$ & $-7.63 \mathrm{E}-03$ & $1.09 \mathrm{E}-03$ \\
\hline & 18 & $1.06 \mathrm{E}-02$ & $3.83 \mathrm{E}-16$ & $2.70 \mathrm{E}-03$ & $2.70 \mathrm{E}-03$ & $-1.55 \mathrm{E}-03$ & $5.48 \mathrm{E}-04$ \\
\hline & 71 & $5.61 \mathrm{E}-02$ & $3.77 \mathrm{E}-16$ & $1.61 \mathrm{E}-02$ & $1.61 \mathrm{E}-02$ & $-1.10 \mathrm{E}-02$ & $1.51 \mathrm{E}-03$ \\
\hline & 74 & $1.27 \mathrm{E}-02$ & $1.92 \mathrm{E}-16$ & $3.53 \mathrm{E}-03$ & $3.53 \mathrm{E}-03$ & $-2.07 \mathrm{E}-03$ & $5.57 \mathrm{E}-04$ \\
\hline \multirow{7}{*}{4} & 46 & $1.52 \mathrm{E}-02$ & $1.27 \mathrm{E}-15$ & $4.03 \mathrm{E}-03$ & $4.03 \mathrm{E}-03$ & $-2.30 \mathrm{E}-03$ & $7.54 \mathrm{E}-04$ \\
\hline & 34 & 2.69E-02 & $1.26 \mathrm{E}-15$ & $7.72 \mathrm{E}-03$ & $7.72 \mathrm{E}-03$ & $-4.46 \mathrm{E}-03$ & $1.13 \mathrm{E}-03$ \\
\hline & 68 & $2.64 \mathrm{E}-02$ & $5.47 \mathrm{E}-16$ & $9.25 \mathrm{E}-03$ & $9.25 \mathrm{E}-03$ & $-5.04 \mathrm{E}-03$ & $7.76 \mathrm{E}-04$ \\
\hline & 32 & $3.37 \mathrm{E}-02$ & $8.21 \mathrm{E}-17$ & $1.08 \mathrm{E}-02$ & $1.08 \mathrm{E}-02$ & $-6.24 \mathrm{E}-03$ & $1.09 \mathrm{E}-03$ \\
\hline & 67 & $1.23 \mathrm{E}-02$ & $6.99 \mathrm{E}-16$ & $3.46 \mathrm{E}-03$ & $3.46 \mathrm{E}-03$ & $-2.00 \mathrm{E}-03$ & $5.38 \mathrm{E}-04$ \\
\hline & 27 & $2.15 \mathrm{E}-02$ & $3.38 \mathrm{E}-15$ & $5.44 \mathrm{E}-03$ & $5.44 \mathrm{E}-03$ & $-3.28 \mathrm{E}-03$ & $1.05 \mathrm{E}-03$ \\
\hline & 83 & $2.38 \mathrm{E}-02$ & $2.15 \mathrm{E}-15$ & $5.55 \mathrm{E}-03$ & $5.55 \mathrm{E}-03$ & $-3.48 \mathrm{E}-03$ & $1.23 \mathrm{E}-03$ \\
\hline \multirow{8}{*}{5} & 59 & $1.50 \mathrm{E}-02$ & $1.16 \mathrm{E}-15$ & $3.95 \mathrm{E}-03$ & $3.95 \mathrm{E}-03$ & $-2.26 \mathrm{E}-03$ & 7.42E-04 \\
\hline & 48 & $2.52 \mathrm{E}-02$ & $1.28 \mathrm{E}-15$ & $7.31 \mathrm{E}-03$ & 7.31E-03 & $-4.16 \mathrm{E}-03$ & $1.07 \mathrm{E}-03$ \\
\hline & 75 & 2.72E-02 & $6.04 \mathrm{E}-16$ & $9.48 \mathrm{E}-03$ & $9.48 \mathrm{E}-03$ & $-5.20 \mathrm{E}-03$ & 7.97E-03 \\
\hline & 91 & $3.34 \mathrm{E}-03$ & 7.64E-16 & $9.86 \mathrm{E}-04$ & $9.86 \mathrm{E}-04$ & $-3.81 \mathrm{E}-04$ & $2.27 \mathrm{E}-04$ \\
\hline & 42 & $3.55 \mathrm{E}-02$ & $1.38 \mathrm{E}-15$ & $1.25 \mathrm{E}-02$ & $1.25 \mathrm{E}-02$ & $-6.90 \mathrm{E}-03$ & $9.89 \mathrm{E}-04$ \\
\hline & 49 & 8.07E-03 & $2.19 \mathrm{E}-15$ & $2.13 \mathrm{E}-03$ & $2.13 \mathrm{E}-03$ & $-1.07 \mathrm{E}-03$ & 4.72E-04 \\
\hline & 29 & $1.58 \mathrm{E}-03$ & $1.64 \mathrm{E}-15$ & $2.78 \mathrm{E}-03$ & $2.78 \mathrm{E}-03$ & $-1.59 \mathrm{E}-03$ & $5.30 \mathrm{E}-04$ \\
\hline & 79 & $1.20 \mathrm{E}-02$ & $4.44 \mathrm{E}-16$ & $3.28 \mathrm{E}-03$ & $3.28 \mathrm{E}-03$ & $-1.95 \mathrm{E}-03$ & $5.28 \mathrm{E}-04$ \\
\hline \multirow{2}{*}{6} & 115 & $2.15 \mathrm{E}-03$ & $1.36 \mathrm{E}-14$ & $5.10 \mathrm{E}-04$ & $5.10 \mathrm{E}-04$ & $-2.12 \mathrm{E}-04$ & $1.62 \mathrm{E}-04$ \\
\hline & 83 & $9.68 \mathrm{E}-03$ & $1.86 \mathrm{E}-15$ & $2.89 \mathrm{E}-03$ & $2.89 \mathrm{E}-03$ & $-1.54 \mathrm{E}-03$ & $4.42 \mathrm{E}-04$ \\
\hline
\end{tabular}




\begin{tabular}{|c|c|c|c|c|c|c|c|}
\hline & 90 & $1.49 \mathrm{E}-02$ & $1.67 \mathrm{E}-15$ & $4.41 \mathrm{E}-03$ & $4.41 \mathrm{E}-03$ & $-2.41 \mathrm{E}-03$ & $6.55 \mathrm{E}-04$ \\
\hline & 81 & 3.09E-02 & $2.94 \mathrm{E}-16$ & $9.53 \mathrm{E}-03$ & $9.53 \mathrm{E}-03$ & $-5.57 \mathrm{E}-03$ & $1.08 \mathrm{E}-03$ \\
\hline & 60 & $1.65 \mathrm{E}-02$ & 7.08E-16 & 4.44E-03 & 4.44E-03 & $-2.66 \mathrm{E}-03$ & 7.31E-04 \\
\hline & 37 & $2.48 \mathrm{E}-02$ & $2.30 \mathrm{E}-15$ & $9.74 \mathrm{E}-03$ & $9.74 \mathrm{E}-03$ & $-4.96 \mathrm{E}-03$ & $6.19 \mathrm{E}-04$ \\
\hline & 50 & $1.70 \mathrm{E}-02$ & $1.46 \mathrm{E}-15$ & $5.02 \mathrm{E}-03$ & $5.02 \mathrm{E}-03$ & $-2.85 \mathrm{E}-03$ & $6.99 \mathrm{E}-04$ \\
\hline & 33 & $1.76 \mathrm{E}-02$ & $9.14 \mathrm{E}-16$ & $5.32 \mathrm{E}-03$ & $5.32 \mathrm{E}-03$ & $-2.81 \mathrm{E}-03$ & 7.95E-04 \\
\hline & 46 & $1.75 \mathrm{E}-02$ & $1.21 \mathrm{E}-15$ & $4.66 \mathrm{E}-03$ & $4.66 \mathrm{E}-03$ & $-2.81 \mathrm{E}-03$ & 7.84E-04 \\
\hline & 59 & $1.03 \mathrm{E}-02$ & $1.78 \mathrm{E}-15$ & $2.92 \mathrm{E}-03$ & $2.92 \mathrm{E}-03$ & $-1.62 \mathrm{E}-03$ & 4.81E-04 \\
\hline \multicolumn{8}{|c|}{ ts9 } \\
\hline \multirow{6}{*}{3} & 33 & $2.29 \mathrm{E}-02$ & $4.62 \mathrm{E}-16$ & $9.08 \mathrm{E}-03$ & $9.08 \mathrm{E}-03$ & $-4.58 \mathrm{E}-03$ & $5.66 \mathrm{E}-04$ \\
\hline & 12 & $2.00 \mathrm{E}-02$ & $4.53 \mathrm{E}-16$ & $4.78 \mathrm{E}-03$ & $4.78 \mathrm{E}-03$ & $-3.00 \mathrm{E}-03$ & $1.00 \mathrm{E}-03$ \\
\hline & 36 & $3.57 \mathrm{E}-02$ & $9.51 \mathrm{E}-15$ & $1.12 \mathrm{E}-02$ & $1.12 \mathrm{E}-02$ & $-6.40 \mathrm{E}-03$ & $1.27 \mathrm{E}-03$ \\
\hline & 61 & 0.1306113 & $1.12 \mathrm{E}-15$ & $2.61 \mathrm{E}-02$ & $2.61 \mathrm{E}-02$ & $-2.48 \mathrm{E}-02$ & $3.92 \mathrm{E}-03$ \\
\hline & 57 & 0.2253427 & 100 & $8.10 \mathrm{E}-02$ & $8.10 \mathrm{E}-02$ & $-9.78 \mathrm{E}-02$ & $-2.08 \mathrm{E}-02$ \\
\hline & 66 & $1.90 \mathrm{E}-02$ & $6.38 \mathrm{E}-16$ & $5.45 \mathrm{E}-03$ & $5.45 \mathrm{E}-03$ & $-2.95 \mathrm{E}-03$ & $9.06 \mathrm{E}-04$ \\
\hline \multirow{3}{*}{4} & 57 & $1.96 \mathrm{E}-02$ & $3.13 \mathrm{E}-15$ & $5.69 \mathrm{E}-03$ & $5.69 \mathrm{E}-03$ & $-3.03 \mathrm{E}-03$ & $9.33 \mathrm{E}-04$ \\
\hline & 52 & 0.1278207 & $3.68 \mathrm{E}-16$ & $2.55 \mathrm{E}-02$ & $2.25 \mathrm{E}-02$ & $-2.40 \mathrm{E}-02$ & 3.96E-03 \\
\hline & 27 & $3.03 \mathrm{E}-02$ & $2.94 \mathrm{E}-16$ & $9.01 \mathrm{E}-03$ & $9.01 \mathrm{E}-03$ & $-5.09 \mathrm{E}-03$ & $1.24 \mathrm{E}-03$ \\
\hline \multirow{6}{*}{5} & 75 & $1.69 \mathrm{E}-02$ & $4.35 \mathrm{E}-16$ & $5.06 \mathrm{E}-03$ & $5.06 \mathrm{E}-03$ & $-2.89 \mathrm{E}-03$ & $6.71 \mathrm{E}-04$ \\
\hline & 48 & 2.91E-02 & $1.16 \mathrm{E}-15$ & $9.84 \mathrm{E}-03$ & $9.84 \mathrm{E}-03$ & $-5.57 \mathrm{E}-03$ & $8.57 \mathrm{E}-04$ \\
\hline & 70 & 0.1215092 & $5.27 \mathrm{E}-16$ & $2.47 \mathrm{E}-02$ & $2.47 \mathrm{E}-02$ & $-2.29 \mathrm{E}-02$ & $3.76 \mathrm{E}-03$ \\
\hline & 50 & $3.34 \mathrm{E}-02$ & $6.40 \mathrm{E}-15$ & $1.06 \mathrm{E}-02$ & $1.06 \mathrm{E}-02$ & $-5.95 \mathrm{E}-03$ & $1.20 \mathrm{E}-03$ \\
\hline & 22 & $1.83 \mathrm{E}-02$ & $2.12 \mathrm{E}-15$ & $4.55 \mathrm{E}-03$ & $4.55 \mathrm{E}-03$ & $-2.91 \mathrm{E}-03$ & 8.37E-04 \\
\hline & 12 & $1.42 \mathrm{E}-02$ & 3.12E-15 & $3.34 \mathrm{E}-03$ & $3.34 \mathrm{E}-03$ & $-2.18 \mathrm{E}-03$ & $6.85 \mathrm{E}-04$ \\
\hline \multirow{6}{*}{6} & 40 & $2.28 \mathrm{E}-02$ & $1.08 \mathrm{E}-15$ & $9.07 \mathrm{E}-03$ & $9.07 \mathrm{E}-03$ & $-4.58 \mathrm{E}-03$ & $5.53 \mathrm{E}-04$ \\
\hline & 25 & $6.38 \mathrm{E}-03$ & $3.98 \mathrm{E}-15$ & $2.16 \mathrm{E}-03$ & $2.16 \mathrm{E}-03$ & $-9.84 \mathrm{E}-04$ & $3.05 \mathrm{E}-04$ \\
\hline & 22 & $1.61 \mathrm{E}-02$ & 2.23E-15 & $4.10 \mathrm{E}-03$ & $4.10 \mathrm{E}-03$ & $-2.39 \mathrm{E}-03$ & 8.13E-04 \\
\hline & 35 & $1.88 \mathrm{E}-02$ & 3.32E-15 & $5.99 \mathrm{E}-03$ & $5.99 \mathrm{E}-03$ & $-3.43 \mathrm{E}-03$ & $6.42 \mathrm{E}-04$ \\
\hline & 57 & 7.53E-02 & $1.18 \mathrm{E}-15$ & $1.39 \mathrm{E}-02$ & $1.39 \mathrm{E}-02$ & $-1.17 \mathrm{E}-02$ & $3.54 \mathrm{E}-03$ \\
\hline & 82 & $2.20 \mathrm{E}-02$ & $1.54 \mathrm{E}-15$ & $6.28 \mathrm{E}-03$ & $6.28 \mathrm{E}-03$ & $-3.53 \mathrm{E}-03$ & $9.83 \mathrm{E}-04$ \\
\hline
\end{tabular}

\section{References:}

(1) Wenz. K. M.; Liu. P.; Houk. K. N. Intramolecular C - H Activation Reactions of Ru(NHC) Complexes Combined with H 2 Transfer to Alkenes: A Theoretical Elucidation of Mechanisms and Effects of Ligands on Reactivities, 2017, 2, https://doi.org/10.1021/acs.organomet.7b00531. 